\title{
Observation of Sequential Y Suppression in PbPb Collisions
}

\author{
S. Chatrchyan et al. ${ }^{*}$ \\ (CMS Collaboration)
}

(Received 13 August 2012; published 26 November 2012)

\begin{abstract}
The suppression of the individual $\mathrm{Y}(n S)$ states in $\mathrm{PbPb}$ collisions with respect to their yields in $p p$ data has been measured. The $\mathrm{PbPb}$ and $p p$ data sets used in the analysis correspond to integrated luminosities of $150 \mu \mathrm{b}^{-1}$ and $230 \mathrm{nb}^{-1}$, respectively, collected in 2011 by the CMS experiment at the LHC, at a center-of-mass energy per nucleon pair of $2.76 \mathrm{TeV}$. The $\mathrm{Y}(n S)$ yields are measured from the dimuon invariant mass spectra. The suppression of the $\mathrm{Y}(n S)$ yields in $\mathrm{PbPb}$ relative to the yields in $p p$, scaled by the number of nucleon-nucleon collisions, $R_{A A}$, is measured as a function of the collision centrality. Integrated over centrality, the $R_{A A}$ values are $0.56 \pm 0.08$ (stat) \pm 0.07 (syst), $0.12 \pm 0.04$ (stat) \pm 0.02 (syst), and lower than 0.10 (at 95\% confidence level), for the $\Upsilon(1 S), \Upsilon(2 S)$, and $\Upsilon(3 S)$ states, respectively. The results demonstrate the sequential suppression of the $\mathrm{Y}(n S)$ states in $\mathrm{PbPb}$ collisions at LHC energies.
\end{abstract}

DOI: 10.1103/PhysRevLett.109.222301

PACS numbers: $25.75 . \mathrm{Nq}, 14.40 . \mathrm{Pq}$

Suppression of heavy quarkonium states has been proposed as a probe of the properties of the hot and dense medium created in high-energy heavy-ion collisions [1]. If a deconfined state, often referred to as the quark-gluon plasma (QGP), is formed, the confining potential of heavy quark-antiquark pairs is expected to be screened because of interactions with quarks and gluons in the medium. The resulting dissociation of the quarkonium states depends on the temperature of the medium, and is expected to occur sequentially, reflecting the increasing values of their binding energies [2]. The $Y(1 S)$ is the most tightly bound quarkonium state, and is hence expected to be the one with the highest dissociation temperature.

The prediction of the suppression pattern is complicated by various factors. These include feed-down contributions from higher-mass resonances into the observed quarkonium yields, as well as several competing nuclear and medium effects. These factors have played an important role in the interpretation of the charmonium measurements [3]. The bottomonium family is expected to provide additional and theoretically cleaner probes of the deconfined medium. The three $Y(n S)$ states, characterized by similar decay kinematics but distinct binding energies, further enable the measurement of relative state suppression, where common experimental and theoretical factors, and respective uncertainties, cancel.

Measurements of the absolute $Y(1 S)$ suppression [4] and of the relative suppression of $\Upsilon(2 S)+\Upsilon(3 S)$ with respect to $Y(1 S)$ [5] were recently reported. These analyses

*Full author list given at the end of the article.

Published by the American Physical Society under the terms of the Creative Commons Attribution 3.0 License. Further distribution of this work must maintain attribution to the author(s) and the published article's title, journal citation, and DOI. used $\mathrm{PbPb}(p p)$ data corresponding to an integrated luminosity of $7.3 \mu \mathrm{b}\left(230 \mathrm{nb}^{-1}\right)$ collected in 2010 (2011) at the same center-of-mass energy per nucleon pair of $\sqrt{s_{N N}}=$ 2.76 TeV, with the Compact Muon Solenoid (CMS) detector at the Large Hadron Collider (LHC). The total $Y$ yields in $\mathrm{PbPb}(p p)$ collisions are denoted by $\left.\mathrm{Y}\right|_{\mathrm{PbPb}}\left(\left.\mathrm{Y}\right|_{p p}\right)$. Selecting reconstructed muons with pseudorapidity $|\eta|<$ 2.4 and transverse momentum $p_{\mathrm{T}}>4 \mathrm{GeV} / c$, the $Y(1 S)$ nuclear modification factor $R_{A A}$ [defined in Eq. (3)] was measured to be $0.63 \pm 0.11$ (stat) \pm 0.10 (syst). The double

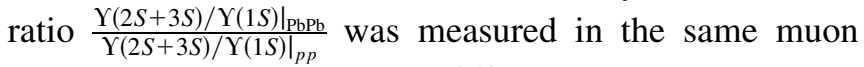
kinematic region to be $0.31_{-0.15}^{+0.19}$ (stat) \pm 0.03 (syst), indicating that the excited $Y(n S)$ states are suppressed with respect to the $Y(1 S)$, at a significance of 2.4 standard deviations $(\sigma)$. In this Letter, an update of these measurements is reported, utilizing a $\mathrm{PbPb}$ data sample corresponding to an integrated luminosity of $150 \mu \mathrm{b}^{-1}$ collected in 2011 by CMS, at $\sqrt{s_{N N}}=2.76 \mathrm{TeV}$ as in the previous study. This larger $\mathrm{PbPb}$ data set together with the excellent momentum resolution of the CMS detector enables the separation of all three $Y$ states below open-bottom threshold in the heavy-ion environment and the measurement of the centrality dependence of their yields.

A detailed description of the CMS detector can be found elsewhere [6]. Its central feature is a superconducting solenoid of $6 \mathrm{~m}$ internal diameter, providing a magnetic field of $3.8 \mathrm{~T}$. Within the field volume are the silicon pixel and strip tracker, the crystal electromagnetic calorimeter, and the brass-scintillator hadron calorimeter. The silicon pixel and strip tracker measures charged-particle trajectories in the range $|\eta|<2.5$. The tracker consists of $66 \mathrm{M}$ pixel and $10 \mathrm{M}$ strip sensor elements. Muons are detected in the range $|\eta|<2.4$, with detection planes based on three technologies: drift tubes, cathode strip chambers, and resistive plate chambers. Because of the strong magnetic field and the fine 
granularity of the tracker, the muon $p_{\mathrm{T}}$ measurement based on information from the tracker alone has a resolution between 1 and $2 \%$ for a typical muon in this analysis.

The CMS apparatus also has extensive forward calorimetry, including two steel-quartz-fiber Čerenkov hadron forward calorimeters (HF), which cover the range $2.9<|\eta|<5.2$. These detectors are used for event selection and centrality determination in $\mathrm{PbPb}$ collisions. The event centrality observable corresponds to the fraction of the total inelastic cross section, starting at $0 \%$ for the most central collisions and evaluated as percentiles of the distribution of the energy deposited in the $\mathrm{HF}[7,8]$. The centrality classes used in this analysis are $50-100 \%$, 40$50 \%, 30-40 \%, 20-30 \%, 10-20 \%, 5-10 \%$, and $0-5 \%$, ordered from the lowest to the highest HF energy deposit. Using a Glauber-model calculation as described in Ref. [7], the average number of nucleons participating in the collisions $\left(N_{\text {part }}\right)$ and the average nuclear overlap function $\left(T_{A A}\right)$ have been estimated for each centrality class. The $T_{A A}$ factor is equal to the number of elementary nucleon-nucleon $(N N)$ binary collisions divided by the elementary $N N$ cross section and can be interpreted as the $N N$-equivalent integrated luminosity per heavy-ion collision, at a given event centrality [9].

The $Y$ states are identified through their dimuon decay. The events are selected online with a hardware-based trigger requiring two muon candidates in the muon detectors. More stringent muon quality requirements are imposed in the $\mathrm{PbPb}$ case relative to the $p p$ online selection. No explicit momentum or rapidity thresholds are applied at trigger level. For the $\mathrm{PbPb}$ data, events are preselected offline if they contain a reconstructed primary vertex comprising at least two tracks, and the presence of energy deposits larger than $3 \mathrm{GeV}$ in at least three towers in each of the two HF calorimeters. These criteria reduce contributions from single-beam interactions, ultraperipheral electromagnetic interactions, and cosmic-ray muons.

Muons are reconstructed by matching tracks in the muon detectors and silicon tracker. The same offline reconstruction algorithm and selection criteria are applied to the $\mathrm{PbPb}$ and $p p$ data samples. The muon candidates are required to have a transverse (longitudinal) distance of closest approach to the event vertex smaller than $3(15) \mathrm{cm}$. Muons are only kept if the part of their trajectory in the tracker has 11 or more hits and the $\chi^{2}$ per degree of freedom of the combined and tracker-only fits is lower than 20 and 4, respectively. Pairs of oppositely charged muons are considered dimuon candidates if the $\chi^{2}$ fit probability of the tracks originating from a common vertex exceeds 5\%. This removes background arising primarily from the displaced, semileptonic decays of charm and bottom hadrons. Only muons with $p_{\mathrm{T}}>4 \mathrm{GeV} / c$ are considered, as in Ref. [5]. The dimuon $p_{\mathrm{T}}$ distribution of the selected candidates extends down to zero and has a mean of about $6 \mathrm{GeV} / c$, covering a dimuon rapidity range of $|y|<$
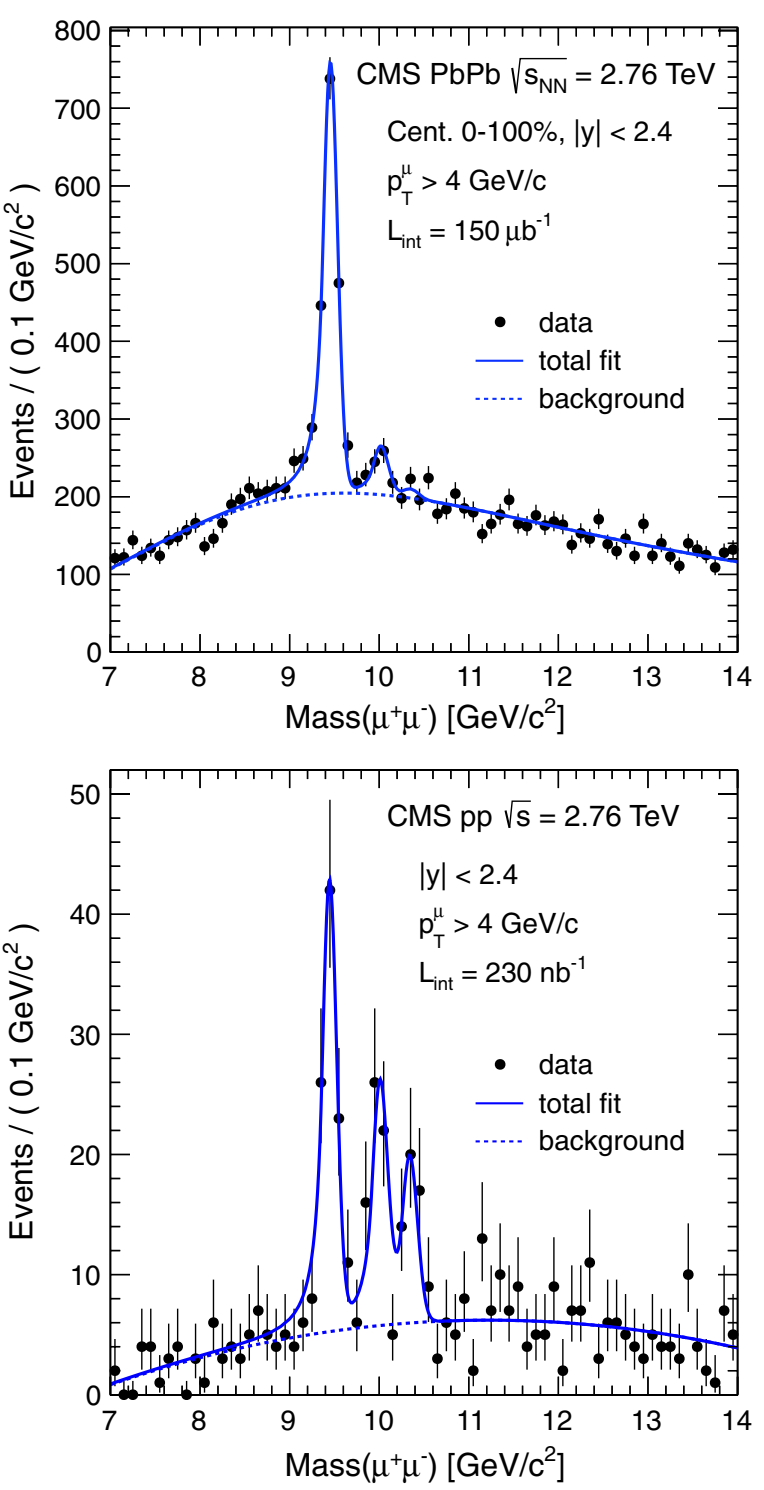

FIG. 1 (color online). Dimuon invariant-mass distributions in $\mathrm{PbPb}$ (top) and $p p$ (bottom) data at $\sqrt{s_{N N}}=2.76 \mathrm{TeV}$. The same reconstruction algorithm and analysis selection are applied to both data sets, including a transverse momentum requirement on single muons of $p_{\mathrm{T}}>4 \mathrm{GeV} / c$. The solid (signal + background) and dashed (background-only) curves show the results of the simultaneous fit to the two data sets.

2.4. The resultant dimuon invariant mass spectra are shown in Fig. 1 for the $\mathrm{PbPb}$ and $p p$ data sets. The three $\mathrm{Y}(n S)$ peaks are clearly observed in the $p p$ case; the $Y(3 S)$ state is not prominent above the dimuon continuum in $\mathrm{PbPb}$ collisions.

Simulated Monte Carlo (MC) events are used to optimize muon selection cuts and to evaluate efficiencies. Signal $Y(n S)$ events are generated using PYTHIA 6.424 [10], with nonrelativistic quantum chromodynamics matrix elements tuned by comparison with CDF data [11]. Underlying heavy-ion events are produced with the 
HYDJET 1.6 [12] event generator. The detector response is simulated with GEANT4 [13]. The signal candidates are embedded in the underlying $\mathrm{PbPb}$ events, at the level of detector hits and with matching vertices. The resulting embedded events are then processed through the trigger emulation and the full event reconstruction chain.

An extended unbinned maximum likelihood fit to the two invariant mass spectra shown in Fig. 1 is performed to extract the $Y(n S)$ yields, following the method described in Refs. [5,14]. The measured mass line shape of each $Y(n S)$ state is parametrized by a "crystal ball" (CB) function, i.e., a Gaussian resolution function with the low-side tail replaced by a power law describing final-state radiation. The mass differences between the states are fixed to their world average values [15] and the mass resolution is forced to scale with the resonance mass. In our previous measurement [5], the signal shape parameters were fixed from MC simulation, including the mass resolution and $\mathrm{CB}$ tail parameters. The current 20 -fold larger $\mathrm{PbPb}$ data set allows these constraints to be released, but the shape parameters are treated as common for both $\mathrm{PbPb}$ and $p p$ data sets via a simultaneous fit.

The background model for the $p p$ data set consists of a second-order polynomial, as was used in Ref. [5], while the larger $\mathrm{PbPb}$ data set requires a more detailed background model. The $p_{\mathrm{T}}>4 \mathrm{GeV} / c$ muon selection threshold causes a depletion of dimuon candidates in the lower part of the $7-14 \mathrm{GeV} / \mathrm{c}^{2}$ mass fitting range. The $\mathrm{PbPb}$ background model consists of an exponential function multiplied by an error function describing the lowmass turn-on. The background parameters are determined from the fit. This nominal model accurately describes the mass sidebands in the opposite-sign muon signal sample, shown in Fig. 1 (top), as well as the alternative estimates of the shape of the combinatorial background obtained from like-sign muon pairs or via a "trackrotation" method. In the latter method [16], the azimuthal angular coordinate of one of the muon tracks is rotated by 180 degrees.

The ratios of the observed yields, not corrected for differences in acceptance and efficiency, of the $Y(2 S)$ and $Y(3 S)$ states to the $Y(1 S)$ state, in the $\mathrm{PbPb}$ and $p p$ data, are

$$
\begin{aligned}
\Upsilon(2 S) /\left.Y(1 S)\right|_{p p}= & 0.56 \pm 0.13 \text { (stat) } \pm 0.02 \text { (syst) } \\
\Upsilon(2 S) /\left.Y(1 S)\right|_{\mathrm{PbPb}}= & 0.12 \pm 0.03 \text { (stat) } \pm 0.02 \text { (syst) } \\
\Upsilon(3 S) /\left.Y(1 S)\right|_{p p}= & 0.41 \pm 0.11(\text { stat }) \pm 0.04 \text { (syst)} \\
\Upsilon(3 S) /\left.Y(1 S)\right|_{\mathrm{PbPb}}= & 0.02 \pm 0.02 \text { (stat) } \pm 0.02 \text { (syst) } \\
& <0.07(95 \% \text { confidence level) }
\end{aligned}
$$

where the systematic uncertainty arises from the fitting procedure, as described below. For the $\mathrm{Y}(3 S)$ to $\mathrm{Y}(1 S)$ ratio in $\mathrm{PbPb}, \mathrm{a} 95 \%$ confidence level (CL) limit is set, based on the Feldman-Cousins statistical method [17].
The measurement of the ratio of the $Y(n S) / \Upsilon(1 S)$ ratios in $\mathrm{PbPb}$ and $p p$ collisions benefits from an almost complete cancellation of possible acceptance or efficiency differences among the reconstructed resonances. The simultaneous fit to the $\mathrm{PbPb}$ and $p p$ mass spectra gives the double ratios

$$
\begin{aligned}
\frac{\Upsilon(2 S) /\left.Y(1 S)\right|_{\mathrm{PbPb}}}{\Upsilon(2 S) /\left.Y(1 S)\right|_{p p}} & =0.21 \pm 0.07 \text { (stat) } \pm 0.02 \text { (syst) } \\
\frac{\Upsilon(3 S) /\left.\Upsilon(1 S)\right|_{\mathrm{PbPb}}}{\Upsilon(3 S) /\left.\Upsilon(1 S)\right|_{p p}} & =0.06 \pm 0.06(\text { stat }) \pm 0.06(\text { syst }) \\
& <0.17(95 \% \mathrm{CL})
\end{aligned}
$$

The systematic uncertainties from the fitting procedure are evaluated by varying the fit function as follows: fixing the $\mathrm{CB}$ tail and resolution parameters to $\mathrm{MC}$ expectations, allowing for differences in these parameters between $\mathrm{PbPb}$ and $p p$, and constraining the background parameters with the like-sign and track-rotated spectra. An additional systematic uncertainty (1\%), estimated from MC simulation, is included to account for possible imperfect cancellations of acceptance and efficiency.

The double ratios, defined in Eq. (2), are expected to be compatible with unity in the absence of suppression of the excited states relative to the $Y(1 S)$ state. The measured values are, instead, considerably smaller than unity. The significance of the observed suppression exceeds $5 \sigma$.

In order to investigate the dependence of the suppression on the centrality of the collision, the double ratio $\frac{\Upsilon(2 S) /\left.Y(1 S)\right|_{p p p b}}{Y(2 S) /\left.Y(1 S)\right|_{p p}}$ is displayed as a function of $N_{\text {part }}$ in Fig. 2 (top) (see the Supplemental Material [18]). The results are constructed from the single ratio $\mathrm{Y}(2 S) /\left.\mathrm{Y}(1 S)\right|_{\mathrm{PbPb}}$ measured in bins of $\mathrm{PbPb}$ centrality, using the $p p$ ratio as normalization. The dependence on centrality is not pronounced. More data, in particular more $p p$ collisions, are needed to establish possible dependences on dimuon kinematic variables.

Absolute suppressions of the individual $Y$ states and their dependence on the collision centrality are studied using the nuclear modification factor, $R_{A A}$, defined as the yield per nucleon-nucleon collision in $\mathrm{PbPb}$ relative to that in $p p$. The $R_{A A}$ observable,

$$
R_{A A}=\frac{\mathcal{L}_{p p}}{T_{A A} N_{\mathrm{MB}}} \frac{\left.\Upsilon(n S)\right|_{\mathrm{PbPb}}}{\left.\Upsilon(n S)\right|_{p p}} \frac{\varepsilon_{p p}}{\varepsilon_{\mathrm{PbPb}}}
$$

is evaluated from the ratio of total $\Upsilon(n S)$ yields in $\mathrm{PbPb}$ and $p p$ collisions corrected for the difference in efficiencies $\varepsilon_{p p} / \varepsilon_{\mathrm{PbPb}}$, with the average nuclear overlap function $T_{A A}$, number of minimum-bias (MB) events sampled by the event selection $N_{\mathrm{MB}}$, and integrated luminosity of the $p p$ data set $\mathcal{L}_{p p}$ accounting for the normalization. The 

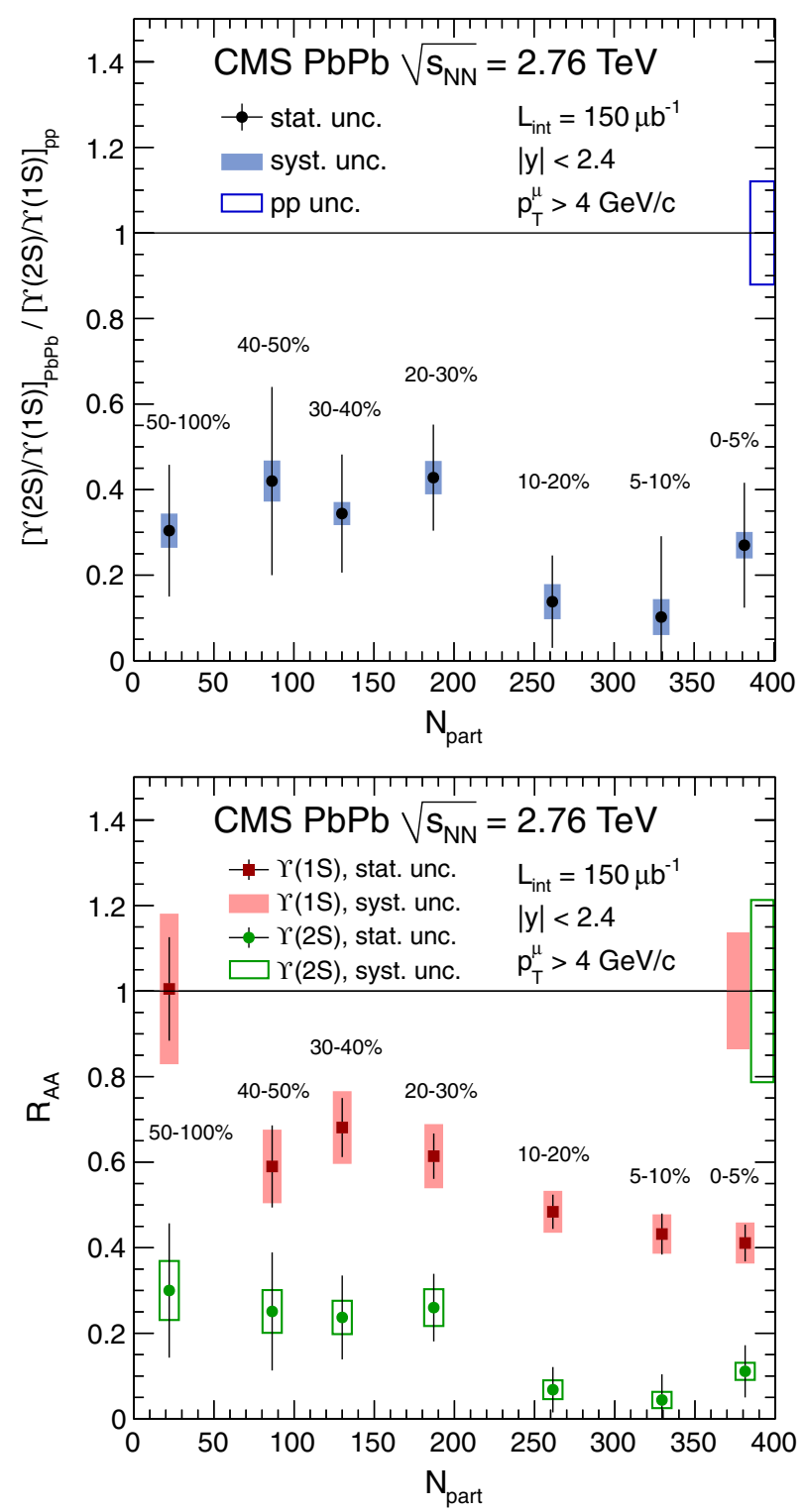

FIG. 2 (color online). Centrality dependence of the double ratio (top) and of the nuclear modification factors (bottom) for the $Y(1 S)$ and $Y(2 S)$ states. The relative uncertainties from $N_{\text {part }}$-independent quantities ( $p p$ yields and, for the $R_{A A}$, also integrated luminosity) are represented by the boxes at unity, and are not included in the data points as these uncertainties do not affect the point-to-point trend. The event centrality bins used are indicated by percentage intervals. The results are available in tabulated form in the Supplemental Material [18].

centrality-integrated (0-100\%) $R_{A A}$ values for the individual $Y$ states are

$$
\begin{aligned}
R_{A A}(Y(1 S))= & 0.56 \pm 0.08(\text { stat }) \pm 0.07 \text { (syst) } \\
R_{A A}(Y(2 S))= & 0.12 \pm 0.04(\text { stat }) \pm 0.02 \text { (syst), } \\
R_{A A}(Y(3 S))= & 0.03 \pm 0.04(\text { stat }) \pm 0.01 \text { (syst) } \\
& <0.10(95 \% \mathrm{CL}) .
\end{aligned}
$$

As the $\mathrm{Y}(3 S)$ peak is not prominent above the dimuon continuum (statistical significance less than 1 standard deviation), an upper limit is also presented. The results for the $Y(1 S)$ and $\Upsilon(2 S)$ obtained by performing the measurement in ranges of centrality are displayed in Fig. 2 (bottom).

Each factor entering in Eq. (3) contributes to the $R_{A A}$ uncertainty, including $\mathcal{L}_{p p}(6 \%)$ and $T_{A A}(4-15 \%$, from central to peripheral collisions). The systematic uncertainties from the fitting procedure, used in the determination of the $Y(1 S)(4-9 \%), Y(2 S)(10-40 \%)$, and $Y(3 S)(14 \%)$ signal yields, are estimated as previously described for the double-ratio measurement. The ratio of efficiencies in Eq. (3) is estimated from MC simulation to deviate by less than $7 \%$ from unity for the centrality bins considered. Systematic uncertainties on the efficiency ratio are estimated by considering variations of simulated kinematic distributions (5-7\%) and from differences in the efficiency ratio estimations from data and $\mathrm{MC}$ simulations (3\%). For the former source, uncertainties are estimated by applying a weight to the generated $Y p_{\mathrm{T}}$ and $|y|$ distributions that increases linearly from 0.7 to 1.3 over the ranges $0<p_{\mathrm{T}}<$ $20 \mathrm{GeV} / c$. For the latter source, reconstruction and trigger selection efficiencies are estimated employing a tag-andprobe method $[4,14]$, using muons from $J / \psi$ decays in $\mathrm{PbPb}$ and $p p$ simulations as well as in collision data.

The results indicate a significant suppression of the $Y(n S)$ states in heavy-ion collisions compared to $p p$ collisions at the same per-nucleon-pair energy. The data support the hypothesis of increased suppression of less strongly bound states: the $Y(1 S)$ is the least suppressed and the $Y(3 S)$ is the most suppressed of the three states. The $\Upsilon(1 S)$ and $\Upsilon(2 S)$ suppressions are observed to increase with collision centrality. The suppression of $\Upsilon(2 S)$ is stronger than that of $\Upsilon(1 S)$ in all centrality ranges, including the most peripheral bin. It should be noted that this bin $(50-100 \%)$ is rather wide and mostly populated by more central events (closer to 50\%). For this most peripheral bin the $Y(1 S)$ nuclear modification factor is $1.01 \pm 0.12$ (stat) \pm 0.22 (syst), while for the most central bin $(0-5 \%) R_{A A}$ is $0.41 \pm 0.04$ (stat) \pm 0.07 (syst) indicating a significant suppression. The observed $Y(n S)$ yields contain contributions from decays of heavier bottomonium states and, thus, the measured suppression is affected by the dissociation of these states. This feeddown contribution to the $Y(1 S)$ state was measured to be of the order of 50\% [19,20], albeit in different kinematic ranges than used here. These results indicate that the directly produced $\Upsilon(1 S)$ state is not significantly suppressed, however quantitative conclusions will require precise estimations of the feed-down contribution matching the phase space of the suppression measurement.

In addition to QGP formation, differences between quarkonium production yields in $\mathrm{PbPb}$ and $p p$ collisions 
can also arise from cold-nuclear-matter effects [21]. However, such effects should have a small impact on the double ratios reported here. Initial-state nuclear effects are expected to affect similarly each of the three $Y$ states, thereby canceling out in the ratio. Final-state "nuclear absorption" becomes weaker with increasing energy [22] and is expected to be negligible at the LHC [23]. Future high-statistics heavy-ion, proton-proton, and protonnucleus runs at the LHC will provide further quarkonium measurements, which will help to disentangle coldnuclear from hot-medium effects and to attain a more thorough characterization of the properties of the produced medium.

In conclusion, the observation of sequential suppression of the $Y(n S)$ states in heavy-ion collisions has been reported, in $\sqrt{s_{N N}}=2.76 \mathrm{TeV} \mathrm{PbPb}$ collisions by the CMS experiment at the LHC, extending the previous CMS bottomonium measurements [4,5]. The $Y(2 S)$ and $\Upsilon(3 S)$ resonances are suppressed with respect to the $Y(1 S)$ state, with a significance exceeding $5 \sigma$. The nuclear modification factors for the $Y(n S)$ states were also measured, with the individual $\mathrm{Y}(1 S), \mathrm{Y}(2 S)$, and $\mathrm{Y}(3 S)$ states suppressed by factors of about 2,8 , and larger than 10 , respectively.

We congratulate our colleagues in the CERN accelerator departments for the excellent performance of the LHC machine. We thank the technical and administrative staff at CERN and other CMS institutes, and acknowledge support from: BMWF and FWF (Austria); FNRS and FWO (Belgium); CNPq, CAPES, FAPERJ, and FAPESP (Brazil); MES (Bulgaria); CERN; CAS, MoST, and NSFC (China); COLCIENCIAS (Colombia); MSES (Croatia); RPF (Cyprus); MEYS (Czech Republic); MoER, SF0690030s09 and ERDF (Estonia); Academy of Finland, MEC, and HIP (Finland); CEA and CNRS/IN2P3 (France); BMBF, DFG, and HGF (Germany); GSRT (Greece); OTKA and NKTH (Hungary); DAE and DST (India); IPM (Iran); SFI (Ireland); INFN (Italy); NRF and WCU (Korea); LAS (Lithuania); CINVESTAV, CONACYT, SEP, and UASLPFAI (Mexico); MSI (New Zealand); PAEC (Pakistan); MSHE and NSC (Poland); FCT (Portugal); JINR (Armenia, Belarus, Georgia, Ukraine, and Uzbekistan); MON, RosAtom, RAS, and RFBR (Russia); MSTD (Serbia); SEIDI and CPAN (Spain); Swiss Funding Agencies (Switzerland); NSC (Taipei); TUBITAK and
TAEK (Turkey); NASU (Ukraine); STFC (United Kingdom); DOE and NSF (USA).

[1] T. Matsui and H. Satz, Phys. Lett. B 178, 416 (1986).

[2] S. Digal, P. Petreczky, and H. Satz, Phys. Rev. D 64, 094015 (2001).

[3] X. Zhao and R. Rapp, Nucl. Phys. A859, 114 (2011).

[4] S. Chatrchyan et al. (CMS Collaboration), J. High Energy Phys. 05 (2012) 063.

[5] S. Chatrchyan et al. (CMS Collaboration), Phys. Rev. Lett. 107, 052302 (2011).

[6] S. Chatrchyan et al. (CMS Collaboration), JINST 03, S08004 (2008).

[7] S. Chatrchyan et al. (CMS Collaboration), Phys. Rev. C 84, 024906 (2011).

[8] D. D'Enterria et al. (CMS Collaboration), J. Phys. G 34, 2307 (2007).

[9] M. L. Miller, K. Reygers, S. J. Sanders, and P. Steinberg, Annu. Rev. Nucl. Part. Sci. 57, 205 (2007).

[10] T. Sjöstrand, S. Mrenna, and P.Z. Skands, J. High Energy Phys. 05 (2006) 026.

[11] D. Acosta et al. (CDF Collaboration), Phys. Rev. D 71, 032001 (2005).

[12] I. P. Lokhtin and A. M. Snigirev, Eur. Phys. J. C 45, 211 (2006).

[13] S. Agostinelli et al. (GEANT4 Collaboration), Nucl. Instrum. Methods Phys. Res., Sect. A 506, 250 (2003).

[14] V. Khachatryan et al. (CMS Collaboration), Phys. Rev. D 83, 112004 (2011).

[15] J. Beringer et al. (Particle Data Group), Phys. Rev. D 86, 010001 (2012).

[16] L. Adamczyk et al. (STAR Collaboration), Phys. Rev. D 86, 072013 (2012).

[17] G. J. Feldman and R. D. Cousins, Phys. Rev. D 57, 3873 (1998).

[18] See Supplemental Material http://link.aps.org/ supplemental/10.1103/PhysRevLett.109.222301 for the centrality-dependent results represented in Fig. 2.

[19] T. Affolder et al. (CDF Collaboration), Phys. Rev. Lett. 84, 2094 (2000).

[20] R. Aaij et al. (LHCb Collaboration), J. High Energy Phys. 11 (2012) 031.

[21] R. Vogt, Phys. Rev. C 81, 044903 (2010).

[22] C. Lourenco, R. Vogt, and H. K. Woehri, J. High Energy Phys. 02 (2009) 014.

[23] Z.-W. Lin and C.M. Ko, Phys. Lett. B 503, 104 (2001).

S. Chatrchyan, ${ }^{1}$ V. Khachatryan, ${ }^{1}$ A. M. Sirunyan, ${ }^{1}$ A. Tumasyan, ${ }^{1}$ W. Adam,${ }^{2}$ E. Aguilo, ${ }^{2}$ T. Bergauer, ${ }^{2}$ M. Dragicevic, ${ }^{2}$ J. Erö, ${ }^{2}$ C. Fabjan, ${ }^{2, b}$ M. Friedl, ${ }^{2}$ R. Frühwirth, ${ }^{2, b}$ V. M. Ghete, ${ }^{2}$ J. Hammer, ${ }^{2}$ N. Hörmann, ${ }^{2}$ J. Hrubec, ${ }^{2}$ M. Jeitler, ${ }^{2, b}$ W. Kiesenhofer, ${ }^{2}$ V. Knünz, ${ }^{2}$ M. Krammer, ${ }^{2, b}$ I. Krätschmer, ${ }^{2}$ D. Liko, ${ }^{2}$ I. Mikulec, ${ }^{2}$ M. Pernicka, ${ }^{2, a}$ B. Rahbaran, ${ }^{2}$ C. Rohringer, ${ }^{2}$ H. Rohringer, ${ }^{2}$ R. Schöfbeck, ${ }^{2}$ J. Strauss, ${ }^{2}$ A. Taurok, ${ }^{2}$ W. Waltenberger, ${ }^{2}$ G. Walzel, ${ }^{2}$ E. Widl, ${ }^{2}$ C.-E. Wulz ${ }^{2, b}$ V. Mossolov, ${ }^{3}$ N. Shumeiko, ${ }^{3}$ J. Suarez Gonzalez, ${ }^{3}$ S. Bansal, ${ }^{4}$ T. Cornelis, ${ }^{4}$ E. A. De Wolf ${ }^{4}$ X. Janssen, ${ }^{4}$ S. Luyckx,${ }^{4}$ L. Mucibello, ${ }^{4}$ S. Ochesanu, ${ }^{4}$ B. Roland, ${ }^{4}$ R. Rougny, ${ }^{4}$ M. Selvaggi, ${ }^{4}$ Z. Staykova, ${ }^{4}$ H. Van Haevermaet, ${ }^{4}$ P. Van Mechelen, ${ }^{4}$ N. Van Remortel,${ }^{4}$ A. Van Spilbeeck, ${ }^{4}$ F. Blekman, ${ }^{5}$ S. Blyweert, ${ }^{5}$ J. D'Hondt, ${ }^{5}$ R. Gonzalez Suarez,${ }^{5}$ A. Kalogeropoulos, ${ }^{5}$ M. Maes, ${ }^{5}$ 
A. Olbrechts, ${ }^{5}$ W. Van Doninck, ${ }^{5}$ P. Van Mulders, ${ }^{5}$ G. P. Van Onsem, ${ }^{5}$ I. Villella, ${ }^{5}$ B. Clerbaux, ${ }^{6}$ G. De Lentdecker, ${ }^{6}$ V. Dero, ${ }^{6}$ A. P. R. Gay, ${ }^{6}$ T. Hreus, ${ }^{6}$ A. Léonard,${ }^{6}$ P. E. Marage, ${ }^{6}$ T. Reis, ${ }^{6}$ L. Thomas, ${ }^{6}$ C. Vander Velde,${ }^{6}$ P. Vanlaer, ${ }^{6}$ J. Wang, ${ }^{6}$ V. Adler, ${ }^{7}$ K. Beernaert, ${ }^{7}$ A. Cimmino, ${ }^{7}$ S. Costantini, ${ }^{7}$ G. Garcia, ${ }^{7}$ M. Grunewald, ${ }^{7}$ B. Klein, ${ }^{7}$ J. Lellouch, ${ }^{7}$ A. Marinov, ${ }^{7}$ J. Mccartin, ${ }^{7}$ A. A. Ocampo Rios ${ }^{7}$ D. Ryckbosch, ${ }^{7}$ N. Strobbe, ${ }^{7}$ F. Thyssen, ${ }^{7}$ M. Tytgat, ${ }^{7}$ P. Verwilligen, ${ }^{7}$ S. Walsh, ${ }^{7}$ E. Yazgan, ${ }^{7}$ N. Zaganidis, ${ }^{7}$ S. Basegmez, ${ }^{8}$ G. Bruno, ${ }^{8}$ R. Castello, ${ }^{8}$ L. Ceard,${ }^{8}$ C. Delaere, ${ }^{8}$ T. du Pree,${ }^{8}$ D. Favart, ${ }^{8}$ L. Forthomme, ${ }^{8}$ A. Giammanco, ${ }^{8, c}$ J. Hollar, ${ }^{8}$ V. Lemaitre, ${ }^{8}$ J. Liao, ${ }^{8}$ O. Militaru, ${ }^{8}$ C. Nuttens, ${ }^{8}$ D. Pagano, ${ }^{8}$ A. Pin, ${ }^{8}$ K. Piotrzkowski, ${ }^{8}$ N. Schul,${ }^{8}$ J. M. Vizan Garcia, ${ }^{8}$ N. Beliy, ${ }^{9}$ T. Caebergs, ${ }^{9}$ E. Daubie, ${ }^{9}$ G. H. Hammad,,${ }^{9}$ G. A. Alves,${ }^{10}$ M. Correa Martins Junior, ${ }^{10}$ D. De Jesus Damiao, ${ }^{10}$ T. Martins,${ }^{10}$ M.E. Pol, ${ }^{10}$ M. H. G. Souza,${ }^{10}$ W.L. Aldá Júnior, ${ }^{11}$ W. Carvalho, ${ }^{11}$ A. Custódio, ${ }^{11}$ E. M. Da Costa,${ }^{11}$

C. De Oliveira Martins,${ }^{11}$ S. Fonseca De Souza, ${ }^{11}$ D. Matos Figueiredo, ${ }^{11}$ L. Mundim, ${ }^{11}$ H. Nogima, ${ }^{11}$ V. Oguri, ${ }^{11}$ W. L. Prado Da Silva, ${ }^{11}$ A. Santoro, ${ }^{11}$ L. Soares Jorge, ${ }^{11}$ A. Sznajder, ${ }^{11}$ T. S. Anjos, ${ }^{12, d}$ C. A. Bernardes, ${ }^{12, d}$ F. A. Dias, ${ }^{12, \mathrm{e}}$ T. R. Fernandez Perez Tomei ${ }^{12}$ E. M. Gregores, ${ }^{12, \mathrm{~d}}$ C. Lagana, ${ }^{12}$ F. Marinho, ${ }^{12}$ P. G. Mercadante,${ }^{12, \mathrm{~d}}$ S. F. Novaes, ${ }^{12}$ Sandra S. Padula, ${ }^{12}$ V. Genchev, ${ }^{13, \mathrm{f}}$ P. Iaydjiev, ${ }^{13, f}$ S. Piperov, ${ }^{13}$ M. Rodozov, ${ }^{13}$ S. Stoykova, ${ }^{13}$ G. Sultanov, ${ }^{13}$ V. Tcholakov, ${ }^{13}$ R. Trayanov, ${ }^{13}$ M. Vutova, ${ }^{13}$ A. Dimitrov, ${ }^{14}$ R. Hadjiiska, ${ }^{14}$ V. Kozhuharov, ${ }^{14}$ L. Litov, ${ }^{14}$ B. Pavlov, ${ }^{14}$ P. Petkov, ${ }^{14}$ J. G. Bian, ${ }^{15}$ G. M. Chen, ${ }^{15}$ H. S. Chen, ${ }^{15}$ C. H. Jiang, ${ }^{15}$ D. Liang, ${ }^{15}$ S. Liang, ${ }^{15}$ X. Meng, ${ }^{15}$ J. Tao, ${ }^{15}$ J. Wang, ${ }^{15}$ X. Wang, ${ }^{15}$ Z. Wang, ${ }^{15}$ H. Xiao, ${ }^{15}$ M. Xu, ${ }^{15}$ J. Zang, ${ }^{15}$ Z. Zhang, ${ }^{15}$

C. Asawatangtrakuldee, ${ }^{16}$ Y. Ban, ${ }^{16}$ S. Guo,${ }^{16}$ Y. Guo,${ }^{16}$ W. Li, ${ }^{16}$ S. Liu, ${ }^{16}$ Y. Mao, ${ }^{16}$ S. J. Qian, ${ }^{16}$ H. Teng, ${ }^{16}$ D. Wang, ${ }^{16}$ L. Zhang, ${ }^{16}$ B. Zhu, ${ }^{16}$ W. Zou, ${ }^{16}$ C. Avila, ${ }^{17}$ J. P. Gomez,${ }^{17}$ B. Gomez Moreno, ${ }^{17}$ A. F. Osorio Oliveros, ${ }^{17}$ J. C. Sanabria,${ }^{17}$ N. Godinovic, ${ }^{18}$ D. Lelas, ${ }^{18}$ R. Plestina, ${ }^{18, \mathrm{~g}}$ D. Polic, ${ }^{18}$ I. Puljak, ${ }^{18, \mathrm{f}}$ Z. Antunovic, ${ }^{19}$ M. Kovac, ${ }^{19}$ V. Brigljevic, ${ }^{20}$ S. Duric, ${ }^{20}$ K. Kadija, ${ }^{20}$ J. Luetic, ${ }^{20}$ S. Morovic, ${ }^{20}$ A. Attikis, ${ }^{21}$ M. Galanti, ${ }^{21}$ G. Mavromanolakis,${ }^{21}$ J. Mousa, ${ }^{21}$ C. Nicolaou, ${ }^{21}$ F. Ptochos,${ }^{21}$ P. A. Razis, ${ }^{21}$ M. Finger,${ }^{22}$ M. Finger, Jr., ${ }^{22}$ Y. Assran, ${ }^{23, \mathrm{~h}}$ S. Elgammal, ${ }^{23, \mathrm{i}}$ A. Ellithi Kamel, ${ }^{23, j}$ S. Khalil, ${ }^{23, i}$ M. A. Mahmoud, ${ }^{23, k}$ A. Radi, ${ }^{23,1, m}$ M. Kadastik, ${ }^{24}$ M. Müntel, ${ }^{24}$ M. Raidal, ${ }^{24}$ L. Rebane, ${ }^{24}$ A. Tiko, ${ }^{24}$ P. Eerola, ${ }^{25}$ G. Fedi, ${ }^{25}$ M. Voutilainen,${ }^{25}$ J. Härkönen ${ }^{26}$ A. Heikkinen ${ }^{26}$ V. Karimäki ${ }^{26}$ R. Kinnunen, ${ }^{26}$ M. J. Kortelainen, ${ }^{26}$ T. Lampén, ${ }^{26}$ K. Lassila-Perini, ${ }^{26}$ S. Lehti, ${ }^{26}$ T. Lindén, ${ }^{26}$ P. Luukka, ${ }^{26}$ T. Mäenpää, ${ }^{26}$ T. Peltola, ${ }^{26}$ E. Tuominen, ${ }^{26}$ J. Tuominiemi, ${ }^{26}$ E. Tuovinen, ${ }^{26}$ D. Ungaro, ${ }^{26}$ L. Wendland, ${ }^{26}$ K. Banzuzi, ${ }^{27}$ A. Karjalainen, ${ }^{27}$ A. Korpela, ${ }^{27}$ T. Tuuva, ${ }^{27}$ M. Besancon, ${ }^{28}$ S. Choudhury, ${ }^{28}$ M. Dejardin, ${ }^{28}$ D. Denegri, ${ }^{28}$ B. Fabbro, ${ }^{28}$ J. L. Faure,${ }^{28}$ F. Ferri, ${ }^{28}$ S. Ganjour ${ }^{28}$ A. Givernaud,${ }^{28}$ P. Gras, ${ }^{28}$

G. Hamel de Monchenault, ${ }^{28}$ P. Jarry, ${ }^{28}$ E. Locci, ${ }^{28}$ J. Malcles, ${ }^{28}$ L. Millischer ${ }^{28}$ A. Nayak, ${ }^{28}$ J. Rander, ${ }^{28}$ A. Rosowsky, ${ }^{28}$ I. Shreyber, ${ }^{28}$ M. Titov, ${ }^{28}$ S. Baffioni, ${ }^{29}$ F. Beaudette, ${ }^{29}$ L. Benhabib,${ }^{29}$ L. Bianchini, ${ }^{29}$ M. Bluj, ${ }^{29, n}$ C. Broutin,${ }^{29}$ P. Busson, ${ }^{29}$ C. Charlot,${ }^{29}$ N. Daci, ${ }^{29}$ T. Dahms, ${ }^{29}$ L. Dobrzynski, ${ }^{29}$ R. Granier de Cassagnac, ${ }^{29}$ M. Haguenauer, ${ }^{29}$ P. Miné, ${ }^{29}$ C. Mironov, ${ }^{29}$ I. N. Naranjo, ${ }^{29}$ M. Nguyen, ${ }^{29}$ C. Ochando, ${ }^{29}$ P. Paganini, ${ }^{29}$ D. Sabes,${ }^{29}$ R. Salerno, ${ }^{29}$ Y. Sirois,${ }^{29}$ C. Veelken,${ }^{29}$ A. Zabi, ${ }^{29}$ J.-L. Agram,${ }^{30, o}$ J. Andrea,${ }^{30}$ D. Bloch, ${ }^{30}$ D. Bodin,${ }^{30}$ J.-M. Brom, ${ }^{30}$ M. Cardaci, ${ }^{30}$ E. C. Chabert, ${ }^{30}$ C. Collard ${ }^{30}$ E. Conte, ${ }^{30, o}$ F. Drouhin, ${ }^{30, o}$ C. Ferro, ${ }^{30}$ J.-C. Fontaine, ${ }^{30, o}$ D. Gelé, ${ }^{30}$ U. Goerlach, ${ }^{30}$ P. Juillot, ${ }^{30}$ A.-C. Le Bihan, ${ }^{30}$ P. Van Hove,${ }^{30}$ F. Fassi, ${ }^{31}$ D. Mercier, ${ }^{31}$ S. Beauceron, ${ }^{32}$ N. Beaupere, ${ }^{32}$ O. Bondu, ${ }^{32}$ G. Boudoul, ${ }^{32}$ J. Chasserat,${ }^{32}$ R. Chierici, ${ }^{32, f}$ D. Contardo,${ }^{32}$ P. Depasse, ${ }^{32}$ H. El Mamouni, ${ }^{32}$ J. Fay, ${ }^{32}$ S. Gascon, ${ }^{32}$ M. Gouzevitch, ${ }^{32}$ B. Ille, ${ }^{32}$ T. Kurca, ${ }^{32}$ M. Lethuillier, ${ }^{32}$ L. Mirabito, ${ }^{32}$ S. Perries, ${ }^{32}$ V. Sordini, ${ }^{32}$ Y. Tschudi, ${ }^{32}$ P. Verdier, ${ }^{32}$ S. Viret, ${ }^{32}$ Z. Tsamalaidze, ${ }^{33, p}$ G. Anagnostou, ${ }^{34}$ S. Beranek,${ }^{34}$ M. Edelhoff, ${ }^{34}$ L. Feld,${ }^{34}$ N. Heracleous,${ }^{34}$ O. Hindrichs, ${ }^{34}$ R. Jussen, ${ }^{34}$ K. Klein, ${ }^{34}$ J. Merz, ${ }^{34}$ A. Ostapchuk,${ }^{34}$ A. Perieanu, ${ }^{34}$ F. Raupach, ${ }^{34}$ J. Sammet, ${ }^{34}$ S. Schael, ${ }^{34}$ D. Sprenger, ${ }^{34}$ H. Weber, ${ }^{34}$ B. Wittmer,${ }^{34}$ V. Zhukov, ${ }^{34, q}$ M. Ata, ${ }^{35}$ J. Caudron, ${ }^{35}$ E. Dietz-Laursonn, ${ }^{35}$ D. Duchardt,${ }^{35}$ M. Erdmann, ${ }^{35}$ R. Fischer, ${ }^{35}$ A. Güth, ${ }^{35}$ T. Hebbeker,${ }^{35}$ C. Heidemann, ${ }^{35}$ K. Hoepfner, ${ }^{35}$ D. Klingebiel,,${ }^{35}$ P. Kreuzer, ${ }^{35}$ C. Magass,${ }^{35}$ M. Merschmeyer, ${ }^{35}$ A. Meyer, ${ }^{35}$ M. Olschewski, ${ }^{35}$ P. Papacz, ${ }^{35}$ H. Pieta, ${ }^{35}$ H. Reithler, ${ }^{35}$ S. A. Schmitz,${ }^{35}$ L. Sonnenschein,${ }^{35}$ J. Steggemann, ${ }^{35}$ D. Teyssier, ${ }^{35}$ M. Weber, ${ }^{35}$ M. Bontenackels, ${ }^{36}$ V. Cherepanov,${ }^{36}$ G. Flügge,${ }^{36}$ H. Geenen, ${ }^{36}$ M. Geisler ${ }^{36}$ W. Haj Ahmad, ${ }^{36}$ F. Hoehle, ${ }^{36}$ B. Kargoll,,${ }^{36}$ T. Kress, ${ }^{36}$ Y. Kuessel, ${ }^{36}$ A. Nowack, ${ }^{36}$ L. Perchalla, ${ }^{36}$ O. Pooth, ${ }^{36}$ P. Sauerland, ${ }^{36}$ A. Stahl, ${ }^{36}$ M. Aldaya Martin, ${ }^{37}$ J. Behr, ${ }^{37}$ W. Behrenhoff, ${ }^{37}$ U. Behrens, ${ }^{37}$ M. Bergholz,,${ }^{37, r}$ A. Bethani, ${ }^{37}$ K. Borras, ${ }^{37}$ A. Burgmeier, ${ }^{37}$ A. Cakir,${ }^{37}$ L. Calligaris, ${ }^{37}$ A. Campbell, ${ }^{37}$ E. Castro, ${ }^{37}$ F. Costanza, ${ }^{37}$ D. Dammann, ${ }^{37}$ C. Diez Pardos,${ }^{37}$ G. Eckerlin, ${ }^{37}$ D. Eckstein, ${ }^{37}$ G. Flucke, ${ }^{37}$ A. Geiser, ${ }^{37}$ I. Glushkov,${ }^{37}$ P. Gunnellini, ${ }^{37}$ S. Habib, ${ }^{37}$ J. Hauk, ${ }^{37}$ G. Hellwig,${ }^{37}$ H. Jung, ${ }^{37}$ M. Kasemann,,${ }^{37}$ P. Katsas, ${ }^{37}$ C. Kleinwort, ${ }^{37}$ H. Kluge, ${ }^{37}$ A. Knutsson, ${ }^{37}$ M. Krämer, ${ }^{37}$ D. Krücker, ${ }^{37}$ E. Kuznetsova, ${ }^{37}$ W. Lange, ${ }^{37}$ W. Lohmann, ${ }^{37, r}$ B. Lutz, ${ }^{37}$ R. Mankel, ${ }^{37}$ I. Marfin, ${ }^{37}$ M. Marienfeld, ${ }^{37}$ I.-A. Melzer-Pellmann, ${ }^{37}$ A. B. Meyer, ${ }^{37}$ J. Mnich, ${ }^{37}$ A. Mussgiller, ${ }^{37}$ S. Naumann-Emme, ${ }^{37}$ J. Olzem, ${ }^{37}$ H. Perrey, ${ }^{37}$ A. Petrukhin,,${ }^{37}$ D. Pitzl, ${ }^{37}$ A. Raspereza, ${ }^{37}$ P. M. Ribeiro Cipriano, ${ }^{37}$ 
C. Riedl,${ }^{37}$ E. Ron, ${ }^{37}$ M. Rosin,,${ }^{37}$ J. Salfeld-Nebgen,${ }^{37}$ R. Schmidt, ${ }^{37, r}$ T. Schoerner-Sadenius,${ }^{37}$ N. Sen, ${ }^{37}$ A. Spiridonov, ${ }^{37}$ M. Stein, ${ }^{37}$ R. Walsh, ${ }^{37}$ C. Wissing, ${ }^{37}$ C. Autermann, ${ }^{38}$ V. Blobel, ${ }^{38}$ J. Draeger,${ }^{38}$ H. Enderle, ${ }^{38}$ J. Erfle, ${ }^{38}$ U. Gebbert, ${ }^{38}$ M. Görner, ${ }^{38}$ T. Hermanns, ${ }^{38}$ R. S. Höing, ${ }^{38}$ K. Kaschube, ${ }^{38}$ G. Kaussen, ${ }^{38}$

H. Kirschenmann, ${ }^{38}$ R. Klanner, ${ }^{38}$ J. Lange, ${ }^{38}$ B. Mura, ${ }^{38}$ F. Nowak,${ }^{38}$ T. Peiffer, ${ }^{38}$ N. Pietsch,${ }^{38}$ D. Rathjens, ${ }^{38}$ C. Sander, ${ }^{38}$ H. Schettler, ${ }^{38}$ P. Schleper, ${ }^{38}$ E. Schlieckau, ${ }^{38}$ A. Schmidt, ${ }^{38}$ M. Schröder, ${ }^{38}$ T. Schum, ${ }^{38}$ M. Seidel, ${ }^{38}$ V. Sola,${ }^{38}$ H. Stadie, ${ }^{38}$ G. Steinbrück, ${ }^{38}$ J. Thomsen, ${ }^{38}$ L. Vanelderen, ${ }^{38}$ C. Barth, ${ }^{39}$ J. Berger, ${ }^{39}$ C. Böser, ${ }^{39}$ T. Chwalek, ${ }^{39}$ W. De Boer, ${ }^{39}$ A. Descroix, ${ }^{39}$ A. Dierlamm, ${ }^{39}$ M. Feindt, ${ }^{39}$ M. Guthoff, $,{ }^{39}, \mathrm{f}$ C. Hackstein, ${ }^{39}$ F. Hartmann, ${ }^{39}$ T. Hauth, ${ }^{39, f}$ M. Heinrich, ${ }^{39}$ H. Held, ${ }^{39}$ K. H. Hoffmann,${ }^{39}$ S. Honc, ${ }^{39}$ I. Katkov, ${ }^{39, q}$ J. R. Komaragiri, ${ }^{39}$ P. Lobelle Pardo, ${ }^{39}$ D. Martschei, ${ }^{39}$ S. Mueller, ${ }^{39}$ Th. Müller, ${ }^{39}$ M. Niegel, ${ }^{39}$ A. Nürnberg, ${ }^{39}$ O. Oberst, ${ }^{39}$ A. Oehler, ${ }^{39}$ J. Ott,${ }^{39}$ G. Quast,${ }^{39}$ K. Rabbertz,${ }^{39}$ F. Ratnikov, ${ }^{39}$ N. Ratnikova, ${ }^{39}$ S. Röcker, ${ }^{39}$ A. Scheurer, ${ }^{39}$ F.-P. Schilling, ${ }^{39}$ G. Schott, ${ }^{39}$ H. J. Simonis, ${ }^{39}$ F. M. Stober, ${ }^{39}$ D. Troendle, ${ }^{39}$ R. Ulrich, ${ }^{39}$ J. Wagner-Kuhr, ${ }^{39}$ S. Wayand, ${ }^{39}$ T. Weiler, ${ }^{39}$ M. Zeise, ${ }^{39}$ G. Daskalakis, ${ }^{40}$ T. Geralis, ${ }^{40}$ S. Kesisoglou,,${ }^{40}$ A. Kyriakis, ${ }^{40}$ D. Loukas, ${ }^{40}$ I. Manolakos, ${ }^{40}$ A. Markou, ${ }^{40}$ C. Markou, ${ }^{40}$ C. Mavrommatis, ${ }^{40}$ E. Ntomari,${ }^{40}$ L. Gouskos, ${ }^{41}$ T. J. Mertzimekis, ${ }^{41}$ A. Panagiotou,${ }^{41}$ N. Saoulidou, ${ }^{41}$ I. Evangelou, ${ }^{42}$ C. Foudas, ${ }^{42, f}$ P. Kokkas, ${ }^{42}$ N. Manthos ${ }^{42}$ I. Papadopoulos, ${ }^{42}$ V. Patras, ${ }^{42}$ G. Bencze, ${ }^{43}$ C. Hajdu,${ }^{43, f}$ P. Hidas, ${ }^{43}$ D. Horvath, ${ }^{43, s}$ F. Sikler, ${ }^{43}$ V. Veszpremi, ${ }^{43}$ G. Vesztergombi, ${ }^{43, t}$ A. Zsigmond, ${ }^{43}$ N. Beni, ${ }^{44}$ S. Czellar, ${ }^{44}$ J. Molnar, ${ }^{44}$ J. Palinkas, ${ }^{44}$ Z. Szillasi ${ }^{44}$ J. Karancsi, ${ }^{45}$ P. Raics,${ }^{45}$ Z. L. Trocsanyi, ${ }^{45}$ B. Ujvari, ${ }^{45}$ S. B. Beri,${ }^{46}$ V. Bhatnagar, ${ }^{46}$ N. Dhingra, ${ }^{46}$ R. Gupta ${ }^{46}$ M. Jindal,${ }^{46}$ M. Kaur, ${ }^{46}$ M.Z. Mehta, ${ }^{46}$ N. Nishu,${ }^{46}$ L. K. Saini, ${ }^{46}$ A. Sharma,${ }^{46}$ J. Singh, ${ }^{46}$ Ashok Kumar, ${ }^{47}$ Arun Kumar, ${ }^{47}$ S. Ahuja ${ }^{47}$ A. Bhardwaj, ${ }^{47}$ B. C. Choudhary ${ }^{47}$ S. Malhotra, ${ }^{47}$ M. Naimuddin, ${ }^{47}$ K. Ranjan, ${ }^{47}$ V. Sharma,${ }^{47}$ R. K. Shivpuri, ${ }^{47}$ S. Banerjee, ${ }^{48}$ S. Bhattacharya,${ }^{48}$ S. Dutta ${ }^{48}$ B. Gomber ${ }^{48}$ Sa. Jain, ${ }^{48}$ Sh. Jain, ${ }^{48}$ R. Khurana ${ }^{48}$ S. Sarkar, ${ }^{48}$ M. Sharan, ${ }^{48}$ A. Abdulsalam, ${ }^{49}$ R. K. Choudhury, ${ }^{49}$ D. Dutta, ${ }^{49}$ S. Kailas, ${ }^{49}$ V. Kumar, ${ }^{49}$ P. Mehta, ${ }^{49}$ A. K. Mohanty, ${ }^{49, f}$ L. M. Pant ${ }^{49}$ P. Shukla, ${ }^{49}$ T. Aziz, ${ }^{50}$ S. Ganguly, ${ }^{50}$ M. Guchait, ${ }^{50, u}$ M. Maity, ${ }^{50, v}$ G. Majumder,${ }^{50}$ K. Mazumdar, ${ }^{50}$ G. B. Mohanty, ${ }^{50}$ B. Parida, ${ }^{50}$ K. Sudhakar,${ }^{50}$ N. Wickramage, ${ }^{50}$ S. Banerjee,${ }^{51}$ S. Dugad ${ }^{51}$ H. Arfaei,${ }^{52}$ H. Bakhshiansohi,${ }^{52, w}$ S. M. Etesami, ${ }^{52, \mathrm{x}}$ A. Fahim, ${ }^{52, w}$ M. Hashemi, ${ }^{52}$ H. Hesari, ${ }^{52}$ A. Jafari, ${ }^{52, w}$ M. Khakzad, ${ }^{52}$ M. Mohammadi Najafabadi, ${ }^{52}$ S. Paktinat Mehdiabadi, ${ }^{52}$ B. Safarzadeh, ${ }^{52, y}$ M. Zeinali, ${ }^{52, x}$ M. Abbrescia, ${ }^{53 a, 53 b}$ L. Barbone, ${ }^{53 a, 53 b}$ C. Calabria, ${ }^{53 a, 53 b, f}$ S. S. Chhibra, ${ }^{53 a, 53 b}$ A. Colaleo, ${ }^{53 a}$ D. Creanza, ${ }^{53 a, 53 c}$ N. De Filippis, ${ }^{53 a, 53 c, f}$ M. De Palma ${ }^{53 a, 53 b}$ L. Fiore, ${ }^{53 a}$ G. Iaselli, ${ }^{53 a, 53 c}$ L. Lusito, ${ }^{53 a, 53 b}$ G. Maggi, ${ }^{53 a, 53 c}$ M. Maggi, ${ }^{53 a}$ B. Marangelli, ${ }^{53 a, 53 b}$ S. My, ${ }^{53 a, 53 c}$ S. Nuzzo, ${ }^{53 a, 53 b}$ N. Pacifico, ${ }^{53 a, 53 b}$ A. Pompili, ${ }^{53 a, 53 b}$ G. Pugliese,${ }^{53 a, 53 c}$ G. Selvaggi, ${ }^{53 a, 53 b}$ L. Silvestris, ${ }^{53 a}$ G. Singh, ${ }^{53 a, 53 b}$ R. Venditti, ${ }^{53 a}$ G. Zito, ${ }^{53 a}$ G. Abbiendi, ${ }^{54 a}$ A. C. Benvenuti, ${ }^{54 a}$ D. Bonacorsi, ${ }^{54 a, 54 b}$ S. Braibant-Giacomelli, ${ }^{54 a, 54 b}$ L. Brigliadori, ${ }^{54 a, 54 b}$ P. Capiluppi, ${ }^{54 a, 54 b}$ A. Castro, ${ }^{54 a, 54 b}$ F. R. Cavallo, ${ }^{54 a}$ M. Cuffiani, ${ }^{54 a, 54 b}$ G. M. Dallavalle, ${ }^{54 a}$ F. Fabbri, ${ }^{54 a}$ A. Fanfani, ${ }^{54 a, 54 b}$ D. Fasanella, ${ }^{54 a, 54 b, f}$ P. Giacomelli, ${ }^{54 a}$ C. Grandi, ${ }^{54 a}$ L. Guiducci, ${ }^{54 a, 54 b}$ S. Marcellini, ${ }^{54 a}$ G. Masetti, ${ }^{54 a}$ M. Meneghelli, ${ }^{54 a, 54 b, f}$ A. Montanari, ${ }^{54 a}$ F. L. Navarria, ${ }^{54 a, 54 b}$ F. Odorici, ${ }^{54 a}$ A. Perrotta, ${ }^{54 a}$ F. Primavera, ${ }^{54 a, 54 b}$ A. M. Rossi, ${ }^{54 a, 54 b}$ T. Rovelli, ${ }^{54 a, 54 b}$ G. Siroli, ${ }^{54 a, 54 b}$ R. Travaglini, ${ }^{54 a, 54 b}$ S. Albergo, ${ }^{55 a, 55 b}$ G. Cappello, ${ }^{55 a, 55 b}$ M. Chiorboli, ${ }^{55 a, 55 b}$ S. Costa, ${ }^{55 a, 55 b}$ R. Potenza, ${ }^{55 a, 55 b}$ A. Tricomi, ${ }^{55 a, 55 b}$ C. Tuve,${ }^{55 a, 55 b}$ G. Barbagli, ${ }^{56 a}$ V. Ciulli, ${ }^{56 a, 56 b}$ C. Civinini, ${ }^{56 a}$ R. D'Alessandro, ${ }^{56 a, 56 b}$ E. Focardi, ${ }^{56 a, 56 b}$ S. Frosali, ${ }^{56 a, 56 b}$ E. Gallo, ${ }^{56 a}$ S. Gonzi, ${ }^{56 a, 56 b}$ M. Meschini ${ }^{56 a}$ S. Paoletti, ${ }^{56 a}$ G. Sguazzoni,${ }^{56 a}$ A. Tropiano, ${ }^{56 a, f}$ L. Benussi, ${ }^{57}$ S. Bianco, ${ }^{57}$ S. Colafranceschi, ${ }^{57, z}$ F. Fabbri, ${ }^{57}$ D. Piccolo, ${ }^{57}$ P. Fabbricatore, ${ }^{58 a}$ R. Musenich, ${ }^{58 a}$ S. Tosi, ${ }^{58 a}$ A. Benaglia,,${ }^{59,59 b, f}$ F. De Guio, ${ }^{59 a, 59 b}$ L. Di Matteo,${ }^{59 a, 59 b, f}$ S. Fiorendi, ${ }^{59 a, 59 b}$ S. Gennai, ${ }^{59 a, f}$ A. Ghezzi, ${ }^{59 a, 59 b}$ S. Malvezzi, ${ }^{59 a}$ R. A. Manzoni, ${ }^{59 a, 59 b}$ A. Martelli, ${ }^{59 a, 59 b}$ A. Massironi, ${ }^{59 a, 59 b, f}$ D. Menasce, ${ }^{59 a}$ L. Moroni, ${ }^{59 a}$ M. Paganoni, ${ }^{59 a, 59 b}$ D. Pedrini, ${ }^{59 a}$ S. Ragazzi,${ }^{59 a, 59 b}$ N. Redaelli, ${ }^{59 a}$ S. Sala ${ }^{59 a}$ T. Tabarelli de Fatis,${ }^{59 a, 59 b}$ S. Buontempo ${ }^{60 a}$ C. A. Carrillo Montoya, ${ }^{60 a}$ N. Cavallo, ${ }^{60 a, a a}$ A. De Cosa, ${ }^{60 a, 60 b, f}$ O. Dogangun, ${ }^{60 a, 60 b}$ F. Fabozzi, ${ }^{60 a, a a}$ A. O. M. Iorio, ${ }^{60 a}$ L. Lista, ${ }^{60 a}$ S. Meola, ${ }^{60, b b}$ M. Merola,${ }^{60 a, 60 b}$ P. Paolucci, ${ }^{60 a, f}$ P. Azzi ${ }^{61 a}$ N. Bacchetta, ${ }^{61 a, f}$ M. Biasotto, ${ }^{61 a, c c}$ D. Bisello, ${ }^{61 a, 61 b}$ A. Branca, ${ }^{61 \mathrm{a}, \mathrm{f}}$ P. Checchia, ${ }^{61 \mathrm{a}}$ T. Dorigo, ${ }^{61 \mathrm{a}}$ F. Gasparini, ${ }^{61 \mathrm{a}, 61 \mathrm{~b}}$ F. Gonella, ${ }^{61 \mathrm{a}}$ A. Gozzelino, ${ }^{61 \mathrm{a}} \mathrm{M}$. Gulmini, ${ }^{61 \mathrm{a}, \mathrm{cc}}$ K. Kanishchev, ${ }^{61 \mathrm{a}, 61 \mathrm{c}}$ S. Lacaprara, ${ }^{61 \mathrm{a}}$ I. Lazzizzera, ${ }^{61 \mathrm{a}, 61 \mathrm{c}}$ M. Margoni, ${ }^{61 \mathrm{a}, 61 \mathrm{~b}}$ G. Maron ${ }^{61 \mathrm{a}, \mathrm{cc}}$

A. T. Meneguzzo, ${ }^{61 \mathrm{a}, 61 \mathrm{~b}}$ F. Montecassiano, ${ }^{61 \mathrm{a}}$ J. Pazzini, ${ }^{61 \mathrm{a}}$ N. Pozzobon, ${ }^{61 \mathrm{a}, 61 \mathrm{~b}}$ P. Ronchese, ${ }^{61 \mathrm{a}, 61 \mathrm{~b}}$ E. Torassa, ${ }^{61 \mathrm{a}}$ M. Tosi, ${ }^{61 a, 61 b, f}$ S. Vanini, ${ }^{61 a, 61 b}$ M. Gabusi, ${ }^{62 a, 62 b}$ S. P. Ratti, ${ }^{62 a, 62 b}$ C. Riccardi, ${ }^{62 a, 62 b}$ P. Torre, ${ }^{62 a, 62 b}$ P. Vitulo, ${ }^{62 a, 62 b}$ M. Biasini, ${ }^{63 a, 63 b}$ G. M. Bilei, ${ }^{63 a}$ L. Fanò, ${ }^{63 a, 63 b}$ P. Lariccia, ${ }^{63 a, 63 b}$ A. Lucaroni, ${ }^{63 a, 63 b, f}$ G. Mantovani, ${ }^{63 a, 63 b}$

M. Menichelli ${ }^{63 a}$ A. Nappi ${ }^{63 a, 63 b}$ F. Romeo, ${ }^{63 a, 63 b}$ A. Saha ${ }^{63 a}$ A. Santocchia,${ }^{63 a, 63 b}$ A. Spiezia, ${ }^{63 a, 63 b}$ S. Taroni, ${ }^{63 a, 63 b, f}$ P. Azzurri, ${ }^{64 a, 64 c}$ G. Bagliesi, ${ }^{64 a}$ T. Boccali, ${ }^{64 a}$ G. Broccolo,${ }^{64 a, 64 c}$ R. Castaldi, ${ }^{64 a}$ R. T. D'Agnolo, ${ }^{64 a, 64 c}$ R. Dell'Orso, ${ }^{64 a}$ F. Fiori, ${ }^{64 a, 64 b, f}$ L. Foà, ${ }^{64 a, 64 c}$ A. Giassi, ${ }^{64 a}$ A. Kraan, ${ }^{64 a}$ F. Ligabue, ${ }^{64 a, 64 c}$ 
T. Lomtadze, ${ }^{64 a}$ L. Martini, ${ }^{64 a, d d}$ A. Messineo,${ }^{64 a, 64 b}$ F. Palla, ${ }^{64 a}$ A. Rizzi, ${ }^{64 a, 64 b}$ A. T. Serban, ${ }^{64 a, e e}$ P. Spagnolo, ${ }^{64 a}$ P. Squillacioti, ${ }^{64 a, f}$ R. Tenchini, ${ }^{64 a}$ G. Tonelli, ${ }^{64 a, 64 b, f}$ A. Venturi, ${ }^{64 a, f}$ P. G. Verdini, ${ }^{64 a}$ L. Barone,${ }^{65 a, 65 b}$ F. Cavallari ${ }^{65 a}$ D. Del Re,${ }^{65 a, 65 b, f}$ M. Diemoz ${ }^{65 a}$ C. Fanelli, ${ }^{65 a}$ M. Grassi,${ }^{65 a, 65 b, f}$ E. Longo ${ }^{65 a, 65 b}$ P. Meridiani, ${ }^{65 a, f}$ F. Micheli, ${ }^{65 a, 65 b}$ S. Nourbakhsh, ${ }^{65 a, 65 b}$ G. Organtini, ${ }^{65 a, 65 b}$ R. Paramatti, ${ }^{65 a}$ S. Rahatlou, ${ }^{65 a, 65 b}$ M. Sigamani, ${ }^{65 a}$ L. Soffi, ${ }^{65 a, 65 b}$ N. Amapane, ${ }^{66 a, 66 b}$ R. Arcidiacono, ${ }^{66 a, 66 \mathrm{c}}$ S. Argiro, ${ }^{66 \mathrm{a}, 66 \mathrm{~b}} \mathrm{M}$. Arneodo, ${ }^{66 \mathrm{a}, 66 \mathrm{c}} \mathrm{C}$. Biino, ${ }^{66 \mathrm{a}} \mathrm{N}$. Cartiglia, ${ }^{66 \mathrm{a}}$ M. Costa, ${ }^{66 a, 66 b}$ N. Demaria, ${ }^{66 a}$ C. Mariotti ${ }^{66 a, f}$ S. Maselli, ${ }^{66 a}$ E. Migliore ${ }^{66 a, 66 b}$ V. Monaco, ${ }^{66 a, 66 b}$ M. Musich,${ }^{66 a, f}$ M. M. Obertino, ${ }^{66 a, 66 c}$ N. Pastrone, ${ }^{66 a}$ M. Pelliccioni, ${ }^{66 a}$ A. Potenza, ${ }^{66 a, 66 b}$ A. Romero, ${ }^{66 a, 66 b}$ R. Sacchi,${ }^{66 a, 66 b}$ A. Solano, ${ }^{66 a, 66 b}$ A. Staiano, ${ }^{66 a}$ P. P. Trapani, ${ }^{66 a, 66 b}$ A. Vilela Pereira, ${ }^{66 a}$ S. Belforte, ${ }^{67 a}$ V. Candelise, ${ }^{67 a, 67 b}$ F. Cossutti, ${ }^{67 a}$ G. Della Ricca, ${ }^{67,67 b}$ B. Gobbo, ${ }^{67 a}$ M. Marone, ${ }^{67 a, 67 b, f}$ D. Montanino, ${ }^{67 a, 67 b, f}$ A. Penzo, ${ }^{67 a}$ A. Schizzi, ${ }^{67 a, 67 b}$ S. G. Heo, ${ }^{68}$ T. Y. Kim, ${ }^{68}$ S. K. Nam, ${ }^{68}$ S. Chang, ${ }^{69}$ D. H. Kim, ${ }^{69}$ G. N. Kim, ${ }^{69}$ D. J. Kong, ${ }^{69}$ H. Park, ${ }^{69}$ S. R. Ro, ${ }^{69}$ D. C. Son, ${ }^{69}$ T. Son ${ }^{69}$ J. Y. Kim, ${ }^{70}$ Zero J. Kim ${ }^{70}$ S. Song,${ }^{70}$ S. Choi, ${ }^{71}$ D. Gyun, ${ }^{71}$ B. Hong, ${ }^{71}$ M. Jo ${ }^{71}$ H. Kim, ${ }^{71}$ T. J. Kim,${ }^{71}$ K. S. Lee,${ }^{71}$ D. H. Moon, ${ }^{71}$ S. K. Park, ${ }^{71}$ M. Choi, ${ }^{72}$ J. H. Kim, ${ }^{72}$ C. Park, ${ }^{72}$ I. C. Park, ${ }^{72}$ S. Park, ${ }^{72}$ G. Ryu,${ }^{72}$ Y. Cho, ${ }^{73}$ Y. Choi, ${ }^{73}$ Y. K. Choi,${ }^{73}$ J. Goh,${ }^{73}$ M. S. Kim, ${ }^{73}$ E. Kwon, ${ }^{73}$ B. Lee, ${ }^{73}$ J. Lee, ${ }^{73}$ S. Lee, ${ }^{73}$ H. Seo, ${ }^{73}$ I. Yu, ${ }^{73}$ M. J. Bilinskas, ${ }^{74}$ I. Grigelionis, ${ }^{74}$ M. Janulis, ${ }^{74}$ A. Juodagalvis,${ }^{74}$ H. Castilla-Valdez,${ }^{75}$ E. De La Cruz-Burelo,${ }^{75}$ I. Heredia-de La Cruz,${ }^{75}$ R. Lopez-Fernandez, ${ }^{75}$ R. Magaña Villalba, ${ }^{75}$ J. Martínez-Ortega ${ }^{75}$ A. Sánchez-Hernández, ${ }^{75}$ L. M. Villasenor-Cendejas,${ }^{75}$ S. Carrillo Moreno, ${ }^{76}$ F. Vazquez Valencia ${ }^{76}$ H. A. Salazar Ibarguen, ${ }^{77}$ E. Casimiro Linares,${ }^{78}$ A. Morelos Pineda, ${ }^{78}$ M. A. Reyes-Santos,${ }^{78}$ D. Krofcheck, ${ }^{79}$ A. J. Bell, ${ }^{80}$ P. H. Butler, ${ }^{80}$ R. Doesburg, ${ }^{80}$ S. Reucroft, ${ }^{80}$ H. Silverwood, ${ }^{80}$ M. Ahmad ${ }^{81}$ M. I. Asghar ${ }^{81}$ H. R. Hoorani, ${ }^{81}$ S. Khalid ${ }^{81}$ W. A. Khan,${ }^{81}$ T. Khurshid ${ }^{81}$ S. Qazi, ${ }^{81}$ M. A. Shah,${ }^{81}$ M. Shoaib, ${ }^{81}$ H. Bialkowska, ${ }^{82}$ B. Boimska, ${ }^{82}$ T. Frueboes, ${ }^{82}$ R. Gokieli, ${ }^{82}$ M. Górski, ${ }^{82}$ M. Kazana, ${ }^{82}$ K. Nawrocki, ${ }^{82}$ K. Romanowska-Rybinska, ${ }^{82}$ M. Szleper, ${ }^{82}$ G. Wrochna, ${ }^{82}$ P. Zalewski, ${ }^{82}$ G. Brona, ${ }^{83} \mathrm{~K}$. Bunkowski, ${ }^{83} \mathrm{M}$. Cwiok, ${ }^{83}$ W. Dominik, ${ }^{83}$ K. Doroba, ${ }^{83}$ A. Kalinowski, ${ }^{83}$ M. Konecki, ${ }^{83}$ J. Krolikowski, ${ }^{83}$ N. Almeida, ${ }^{84}$ P. Bargassa ${ }^{84}$

A. David,${ }^{84}$ P. Faccioli ${ }^{84}$ P. G. Ferreira Parracho,${ }^{84}$ M. Gallinaro, ${ }^{84}$ J. Seixas ${ }^{84}$ J. Varela, ${ }^{84}$ P. Vischia,${ }^{84}$ S. Afanasiev, ${ }^{85}$ I. Belotelov, ${ }^{85}$ P. Bunin, ${ }^{85}$ M. Gavrilenko, ${ }^{85}$ I. Golutvin, ${ }^{85}$ A. Kamenev, ${ }^{85}$ V. Karjavin, ${ }^{85}$ G. Kozlov, ${ }^{85}$ A. Lanev, ${ }^{85}$ A. Malakhov, ${ }^{85}$ P. Moisenz, ${ }^{85}$ V. Palichik, ${ }^{85}$ V. Perelygin, ${ }^{85}$ S. Shmatov ${ }^{85}$ V. Smirnov, ${ }^{85}$ A. Volodko, ${ }^{85}$ A. Zarubin, ${ }^{85}$ S. Evstyukhin, ${ }^{86}$ V. Golovtsov, ${ }^{86}$ Y. Ivanov, ${ }^{86}$ V. Kim, ${ }^{86}$ P. Levchenko, ${ }^{86}$ V. Murzin, ${ }^{86}$ V. Oreshkin, ${ }^{86}$ I. Smirnov, ${ }^{86}$ V. Sulimov, ${ }^{86}$ L. Uvarov, ${ }^{86}$ S. Vavilov, ${ }^{86}$ A. Vorobyev, ${ }^{86}$ An. Vorobyev, ${ }^{86}$ Yu. Andreev, ${ }^{87}$ A. Dermenev, ${ }^{87}$ S. Gninenko, ${ }^{87}$ N. Golubev, ${ }^{87}$ M. Kirsanov, ${ }^{87}$ N. Krasnikov, ${ }^{87}$ V. Matveev, ${ }^{87}$ A. Pashenkov, ${ }^{87}$ D. Tlisov, ${ }^{87}$ A. Toropin, ${ }^{87}$ V. Epshteyn,${ }^{88}$ M. Erofeeva, ${ }^{88}$ V. Gavrilov,${ }^{88}$ M. Kossov, ${ }^{88, f}$ N. Lychkovskaya ${ }^{88}$ V. Popov, ${ }^{88}$ G. Safronov, ${ }^{88}$ S. Semenov, ${ }^{88}$ V. Stolin,${ }^{88}$ E. Vlasov ${ }^{88}$ A. Zhokin, ${ }^{88}$ A. Belyaev, ${ }^{89}$ E. Boos, ${ }^{89}$ A. Ershov, ${ }^{89}$

A. Gribushin, ${ }^{89}$ V. Klyukhin, ${ }^{89}$ O. Kodolova ${ }^{89}$ V. Korotkikh, ${ }^{89}$ I. Lokhtin ${ }^{89}$ A. Markina, ${ }^{89}$ S. Obraztsov, ${ }^{89}$ M. Perfilov, ${ }^{89}$ S. Petrushanko, ${ }^{89}$ A. Popov, ${ }^{89}$ L. Sarycheva, ${ }^{89}$ a V. Savrin,${ }^{89}$ A. Snigirev, ${ }^{89}$ I. Vardanyan,${ }^{89}$ V. Andreev, ${ }^{90}$ M. Azarkin, ${ }^{90}$ I. Dremin, ${ }^{90}$ M. Kirakosyan, ${ }^{90}$ A. Leonidov,${ }^{90}$ G. Mesyats, ${ }^{90}$ S. V. Rusakov, ${ }^{90}$ A. Vinogradov, ${ }^{90}$ I. Azhgirey, ${ }^{91}$ I. Bayshev, ${ }^{91}$ S. Bitioukov, ${ }^{91}$ V. Grishin,,${ }^{91, f}$ V. Kachanov, ${ }^{91}$ D. Konstantinov,${ }^{91}$ A. Korablev, ${ }^{91}$ V. Krychkine, ${ }^{91}$ V. Petrov,${ }^{91}$ R. Ryutin, ${ }^{91}$ A. Sobol,${ }^{91}$ L. Tourtchanovitch, ${ }^{91}$ S. Troshin,${ }^{91}$ N. Tyurin, ${ }^{91}$

A. Uzunian, ${ }^{91}$ A. Volkov, ${ }^{91}$ P. Adzic, ${ }^{92, f f}$ M. Djordjevic, ${ }^{92}$ M. Ekmedzic, ${ }^{92}$ D. Krpic,${ }^{92, f f}$ J. Milosevic, ${ }^{92}$ M. Aguilar-Benitez, ${ }^{93}$ J. Alcaraz Maestre,${ }^{93}$ P. Arce, ${ }^{93}$ C. Battilana, ${ }^{93}$ E. Calvo, ${ }^{93}$ M. Cerrada,${ }^{93}$ M. Chamizo Llatas, ${ }^{93}$ N. Colino, ${ }^{93}$ B. De La Cruz, ${ }^{93}$ A. Delgado Peris,${ }^{93}$ D. Domínguez Vázquez, ${ }^{93}$ C. Fernandez Bedoya,${ }^{93}$ J. P. Fernández Ramos, ${ }^{93}$ A. Ferrando, ${ }^{93}$ J. Flix ${ }^{93}$ M. C. Fouz ${ }^{93}$ P. Garcia-Abia,${ }^{93}$ O. Gonzalez Lopez, ${ }^{93}$ S. Goy Lopez, ${ }^{93}$ J. M. Hernandez, ${ }^{93}$ M. I. Josa, ${ }^{93}$ G. Merino, ${ }^{93}$ J. Puerta Pelayo, ${ }^{93}$ A. Quintario Olmeda, ${ }^{93}$ I. Redondo, ${ }^{93}$ L. Romero, ${ }^{93}$ J. Santaolalla, ${ }^{93}$ M. S. Soares, ${ }^{93}$ C. Willmott, ${ }^{93}$ C. Albajar,${ }^{94}$ G. Codispoti, ${ }^{94}$ J. F. de Trocóniz, ${ }^{94}$ H. Brun,,${ }^{95}$ J. Cuevas, ${ }^{95}$ J. Fernandez Menendez, ${ }^{95}$ S. Folgueras, ${ }^{95}$ I. Gonzalez Caballero, ${ }^{95}$ L. Lloret Iglesias, ${ }^{95}$ J. Piedra Gomez, ${ }^{95}$ J. A. Brochero Cifuentes, ${ }^{96}$ I. J. Cabrillo, ${ }^{96}$ A. Calderon, ${ }^{96}$ S. H. Chuang, ${ }^{96}$ J. Duarte Campderros, ${ }^{96}$ M. Felcini, ${ }^{96, g g}$ M. Fernandez, ${ }^{96}$ G. Gomez, ${ }^{96}$ J. Gonzalez Sanchez, ${ }^{96}$ A. Graziano, ${ }^{96}$ C. Jorda,${ }^{96}$ A. Lopez Virto, ${ }^{96}$ J. Marco,${ }^{96}$ R. Marco, ${ }^{96}$ C. Martinez Rivero, ${ }^{96}$ F. Matorras, ${ }^{96}$ F. J. Munoz Sanchez, ${ }^{96}$ T. Rodrigo, ${ }^{96}$ A. Y. Rodríguez-Marrero, ${ }^{96}$ A. Ruiz-Jimeno, ${ }^{96}$ L. Scodellaro, ${ }^{96}$ M. Sobron Sanudo, ${ }^{96}$ I. Vila, ${ }^{96}$ R. Vilar Cortabitarte, ${ }^{96}$ D. Abbaneo, ${ }^{97}$ E. Auffray,${ }^{97}$ G. Auzinger,${ }^{97}$ P. Baillon, ${ }^{97}$ A. H. Ball, ${ }^{97}$ D. Barney, ${ }^{97}$ J. F. Benitez, ${ }^{97}$ C. Bernet, ${ }^{97,9}$ G. Bianchi, ${ }^{97}$ P. Bloch, ${ }^{97}$ A. Bocci, ${ }^{97}$ A. Bonato, ${ }^{97}$ C. Botta, ${ }^{97}$ H. Breuker,${ }^{97}$ T. Camporesi,${ }^{97}$ G. Cerminara, ${ }^{97}$ T. Christiansen, ${ }^{97}$ J. A. Coarasa Perez,${ }^{97}$ D. D'Enterria, ${ }^{97}$

A. Dabrowski, ${ }^{97}$ A. De Roeck, ${ }^{97}$ S. Di Guida, ${ }^{97}$ M. Dobson, ${ }^{97}$ N. Dupont-Sagorin,,${ }^{97}$ A. Elliott-Peisert, ${ }^{97}$ B. Frisch, ${ }^{97}$ W. Funk,${ }^{97}$ G. Georgiou, ${ }^{97}$ M. Giffels,${ }^{97}$ D. Gigi,${ }^{97}$ K. Gill,${ }^{97}$ D. Giordano, ${ }^{97}$ M. Giunta, ${ }^{97}$ F. Glege,${ }^{97}$ 
R. Gomez-Reino Garrido, ${ }^{97}$ P. Govoni, ${ }^{97}$ S. Gowdy, ${ }^{97}$ R. Guida, ${ }^{97}$ M. Hansen, ${ }^{97}$ P. Harris, ${ }^{97}$ C. Hartl, ${ }^{97}$ J. Harvey, ${ }^{97}$

B. Hegner, ${ }^{97}$ A. Hinzmann, ${ }^{97}$ V. Innocente, ${ }^{97}$ P. Janot, ${ }^{97}$ K. Kaadze,${ }^{97}$ E. Karavakis, ${ }^{97}$ K. Kousouris, ${ }^{97}$ P. Lecoq,${ }^{97}$ Y.-J. Lee, ${ }^{97}$ P. Lenzi,${ }^{97}$ C. Lourenço, ${ }^{97}$ T. Mäki,${ }^{97}$ M. Malberti, ${ }^{97}$ L. Malgeri, ${ }^{97}$ M. Mannelli, ${ }^{97}$ L. Masetti, ${ }^{97}$

F. Meijers ${ }^{97}$ S. Mersi, ${ }^{97}$ E. Meschi, ${ }^{97}$ R. Moser, ${ }^{97}$ M. U. Mozer, ${ }^{97}$ M. Mulders,${ }^{97}$ P. Musella, ${ }^{97}$ E. Nesvold, ${ }^{97}$ T. Orimoto, ${ }^{97}$ L. Orsini, ${ }^{97}$ E. Palencia Cortezon, ${ }^{97}$ E. Perez, ${ }^{97}$ L. Perrozzi, ${ }^{97}$ A. Petrilli, ${ }^{97}$ A. Pfeiffer, ${ }^{97}$ M. Pierini, ${ }^{97}$ M. Pimiä, ${ }^{97}$ D. Piparo, ${ }^{97}$ G. Polese,${ }^{97}$ L. Quertenmont, ${ }^{97}$ A. Racz, ${ }^{97}$ W. Reece, ${ }^{97}$ J. Rodrigues Antunes, ${ }^{97}$ G. Rolandi, ${ }^{97, \text { hh }}$ T. Rommerskirchen, ${ }^{97}$ C. Rovelli, ${ }^{97, \text { ii }}$ M. Rovere, ${ }^{97}$ H. Sakulin, ${ }^{97}$ F. Santanastasio, ${ }^{97}$ C. Schäfer, ${ }^{97}$ C. Schwick, ${ }^{97}$ I. Segoni, ${ }^{97}$ S. Sekmen,,${ }^{97}$ A. Sharma, ${ }^{97}$ P. Siegrist, ${ }^{97}$ P. Silva, ${ }^{97}$ M. Simon, ${ }^{97}$ P. Sphicas, ${ }^{97, j j}$ D. Spiga, ${ }^{97}$ A. Tsirou, ${ }^{97}$ G. I. Veres, ${ }^{97, t}$ J. R. Vlimant, ${ }^{97}$ H. K. Wöhri, ${ }^{97}$ S. D. Worm,${ }^{97, k k}$ W. D. Zeuner,${ }^{97}$ W. Bertl, ${ }^{98}$ K. Deiters,${ }^{98}$

W. Erdmann, ${ }^{98}$ K. Gabathuler, ${ }^{98}$ R. Horisberger, ${ }^{98}$ Q. Ingram, ${ }^{98}$ H. C. Kaestli, ${ }^{98}$ S. König, ${ }^{98}$ D. Kotlinski,,${ }^{98}$

U. Langenegger, ${ }^{98}$ F. Meier, ${ }^{98}$ D. Renker,${ }^{98}$ T. Rohe, ${ }^{98}$ J. Sibille, ${ }^{98,11}$ L. Bäni,${ }^{99}$ P. Bortignon, ${ }^{99}$ M. A. Buchmann, ${ }^{99}$ B. Casal, ${ }^{99}$ N. Chanon, ${ }^{99}$ A. Deisher,${ }^{99}$ G. Dissertori,${ }^{99}$ M. Dittmar,${ }^{99}$ M. Donegà, ${ }^{99}$ M. Dünser,${ }^{99}$ J. Eugster, ${ }^{99}$ K. Freudenreich, ${ }^{99}$ C. Grab, ${ }^{99}$ D. Hits, ${ }^{99}$ P. Lecomte, ${ }^{99}$ W. Lustermann, ${ }^{99}$ A. C. Marini, ${ }^{99}$

P. Martinez Ruiz del Arbol, ${ }^{99}$ N. Mohr, ${ }^{99}$ F. Moortgat, ${ }^{99}$ C. Nägeli, ${ }^{99}, \mathrm{~mm}$ P. Nef, ${ }^{99}$ F. Nessi-Tedaldi, ${ }^{99}$ F. Pandolfi, ${ }^{99}$ L. Pape, ${ }^{99}$ F. Pauss, ${ }^{99}$ M. Peruzzi, ${ }^{99}$ F. J. Ronga, ${ }^{99}$ M. Rossini, ${ }^{99}$ L. Sala, ${ }^{99}$ A. K. Sanchez, ${ }^{99}$ A. Starodumov, ${ }^{99, n n}$ B. Stieger, ${ }^{99}$ M. Takahashi, ${ }^{99}$ L. Tauscher, ${ }^{99, a}$ A. Thea, ${ }^{99}$ K. Theofilatos, ${ }^{99}$ D. Treille, ${ }^{99}$ C. Urscheler, ${ }^{99}$ R. Wallny, ${ }^{99}$ H. A. Weber, ${ }^{99}$ L. Wehrli, ${ }^{99}$ C. Amsler, ${ }^{100}$ V. Chiochia, ${ }^{100}$ S. De Visscher, ${ }^{100}$ C. Favaro, ${ }^{100}$ M. Ivova Rikova, ${ }^{100}$ B. Millan Mejias, ${ }^{100}$ P. Otiougova, ${ }^{100}$ P. Robmann, ${ }^{100}$ H. Snoek, ${ }^{100}$ S. Tupputi, ${ }^{100}$ M. Verzetti, ${ }^{100}$ Y. H. Chang, ${ }^{101}$ K. H. Chen, ${ }^{101}$ C. M. Kuo, ${ }^{101}$ S. W. Li,${ }^{101}$ W. Lin, ${ }^{101}$ Z. K. Liu, ${ }^{101}$ Y. J. Lu, ${ }^{101}$ D. Mekterovic, ${ }^{101}$ A. P. Singh, ${ }^{101}$ R. Volpe ${ }^{101}$ S. S. Yu, ${ }^{101}$ P. Bartalini, ${ }^{102}$ P. Chang, ${ }^{102}$ Y. H. Chang, ${ }^{102}$ Y. W. Chang, ${ }^{102}$ Y. Chao, ${ }^{102}$ K. F. Chen, ${ }^{102}$ C. Dietz, ${ }^{102}$ U. Grundler, ${ }^{102}$ W.-S. Hou, ${ }^{102}$ Y. Hsiung, ${ }^{102}$ K. Y. Kao, ${ }^{102}$ Y. J. Lei, ${ }^{102}$ R.-S. Lu, ${ }^{102}$ D. Majumder, ${ }^{102}$ E. Petrakou ${ }^{102}$ X. Shi, ${ }^{102}$ J. G. Shiu,${ }^{102}$ Y. M. Tzeng, ${ }^{102}$ X. Wan, ${ }^{102}$ M. Wang, ${ }^{102}$ A. Adiguzel,${ }^{103}$ M. N. Bakirci, ${ }^{103, \text { oo }}$ S. Cerci, ${ }^{103, p p}$ C. Dozen, ${ }^{103}$ I. Dumanoglu, ${ }^{103}$ E. Eskut, ${ }^{103}$ S. Girgis, ${ }^{103}$ G. Gokbulut, ${ }^{103}$ E. Gurpinar, ${ }^{103}$ I. Hos, ${ }^{103}$

E. E. Kangal, ${ }^{103}$ T. Karaman, ${ }^{103}$ G. Karapinar, ${ }^{103, q 9}$ A. Kayis Topaksu, ${ }^{103}$ G. Onengut, ${ }^{103}$ K. Ozdemir, ${ }^{103}$ S. Ozturk, ${ }^{103, \mathrm{rr}}$ A. Polatoz, ${ }^{103}$ K. Sogut, ${ }^{103, \mathrm{ss}}$ D. Sunar Cerci, ${ }^{103, p p}$ B. Tali, ${ }^{103, p p}$ H. Topakli, ${ }^{103, \text { oo }}$ L. N. Vergili, ${ }^{103}$ M. Vergili, ${ }^{103}$ I. V. Akin, ${ }^{104}$ T. Aliev, ${ }^{104}$ B. Bilin, ${ }^{104}$ S. Bilmis, ${ }^{104}$ M. Deniz, ${ }^{104}$ H. Gamsizkan, ${ }^{104}$ A. M. Guler, ${ }^{104}$ K. Ocalan, ${ }^{104}$ A. Ozpineci, ${ }^{104}$ M. Serin, ${ }^{104}$ R. Sever, ${ }^{104}$ U. E. Surat,${ }^{104}$ M. Yalvac, ${ }^{104}$ E. Yildirim, ${ }^{104}$ M. Zeyrek, ${ }^{104}$ E. Gülmez, ${ }^{105}$ B. Isildak, ${ }^{105, \mathrm{tt}}$ M. Kaya, ${ }^{105, \text { uu }}$ O. Kaya, ${ }^{105, \text { uu }}$ S. Ozkorucuklu, ${ }^{105, \mathrm{vv}}$ N. Sonmez, ${ }^{105, \text { ww }}$ K. Cankocak, ${ }^{106}$ L. Levchuk, ${ }^{107}$ F. Bostock, ${ }^{108}$ J. J. Brooke, ${ }^{108}$ E. Clement, ${ }^{108}$ D. Cussans, ${ }^{108}$ H. Flacher, ${ }^{108}$ R. Frazier,${ }^{108}$

J. Goldstein, ${ }^{108}$ M. Grimes, ${ }^{108}$ G. P. Heath, ${ }^{108}$ H. F. Heath, ${ }^{108}$ L. Kreczko, ${ }^{108}$ S. Metson, ${ }^{108}$ D. M. Newbold,${ }^{108, k k}$ K. Nirunpong, ${ }^{108}$ A. Poll, ${ }^{108}$ S. Senkin, ${ }^{108}$ V. J. Smith, ${ }^{108}$ T. Williams, ${ }^{108}$ L. Basso, ${ }^{109, x x}$ A. Belyaev, ${ }^{109, x x}$ C. Brew, ${ }^{109}$ R. M. Brown, ${ }^{109}$ D. J. A. Cockerill, ${ }^{109}$ J. A. Coughlan, ${ }^{109}$ K. Harder, ${ }^{109}$ S. Harper,${ }^{109}$ J. Jackson, ${ }^{109}$ B. W. Kennedy, ${ }^{109}$ E. Olaiya, ${ }^{109}$ D. Petyt,${ }^{109}$ B. C. Radburn-Smith, ${ }^{109}$ C. H. Shepherd-Themistocleous, ${ }^{109}$ I. R. Tomalin, ${ }^{109}$

W. J. Womersley, ${ }^{109}$ R. Bainbridge, ${ }^{110}$ G. Ball, ${ }^{110}$ R. Beuselinck, ${ }^{110}$ O. Buchmuller, ${ }^{110}$ D. Colling, ${ }^{110}$ N. Cripps,${ }^{110}$ M. Cutajar, ${ }^{110}$ P. Dauncey, ${ }^{110}$ G. Davies,${ }^{110}$ M. Della Negra, ${ }^{110}$ W. Ferguson, ${ }^{110}$ J. Fulcher, ${ }^{110}$ D. Futyan, ${ }^{110}$ A. Gilbert, ${ }^{110}$ A. Guneratne Bryer, ${ }^{110}$ G. Hall,${ }^{110}$ Z. Hatherell, ${ }^{110}$ J. Hays, ${ }^{110}$ G. Iles, ${ }^{110}$ M. Jarvis, ${ }^{110}$

G. Karapostoli, ${ }^{110}$ L. Lyons, ${ }^{110}$ A.-M. Magnan, ${ }^{110}$ J. Marrouche, ${ }^{110}$ B. Mathias, ${ }^{110}$ R. Nandi, ${ }^{110}$ J. Nash,,${ }^{110}$ A. Nikitenko, ${ }^{110, \mathrm{nn}}$ A. Papageorgiou, ${ }^{110}$ J. Pela,${ }^{10, \mathrm{f}}$ M. Pesaresi,${ }^{10}$ K. Petridis, ${ }^{110}$ M. Pioppi,${ }^{110, y y}$

D. M. Raymond,${ }^{110}$ S. Rogerson, ${ }^{110}$ A. Rose, ${ }^{110}$ M. J. Ryan, ${ }^{110}$ C. Seez, ${ }^{110}$ P. Sharp, ${ }^{110, a}$ A. Sparrow, ${ }^{110}$ M. Stoye,${ }^{110}$ A. Tapper, ${ }^{110}$ M. Vazquez Acosta, ${ }^{110}$ T. Virdee, ${ }^{110}$ S. Wakefield, ${ }^{110}$ N. Wardle, ${ }^{110}$ T. Whyntie,${ }^{110}$ M. Chadwick,,${ }^{111}$ J. E. Cole, ${ }^{111}$ P. R. Hobson, ${ }^{111}$ A. Khan, ${ }^{111}$ P. Kyberd, ${ }^{111}$ D. Leggat, ${ }^{111}$ D. Leslie, ${ }^{111}$ W. Martin, ${ }^{111}$ I. D. Reid,,${ }^{111}$ P. Symonds, ${ }^{111}$ L. Teodorescu, ${ }^{111}$ M. Turner, ${ }^{111}$ K. Hatakeyama ${ }^{112}$ H. Liu, ${ }^{112}$ T. Scarborough,,${ }^{112}$ O. Charaf, ${ }^{113}$

C. Henderson, ${ }^{113}$ P. Rumerio, ${ }^{113}$ A. Avetisyan, ${ }^{14}$ T. Bose,${ }^{114}$ C. Fantasia, ${ }^{114}$ A. Heister, ${ }^{114}$ J. St. John, ${ }^{114}$ P. Lawson, ${ }^{114}$ D. Lazic, ${ }^{114}$ J. Rohlf, ${ }^{114}$ D. Sperka, ${ }^{114}$ L. Sulak, ${ }^{114}$ J. Alimena, ${ }^{115}$ S. Bhattacharya, ${ }^{115}$ D. Cutts, ${ }^{115}$ A. Ferapontov, ${ }^{115}$ U. Heintz, ${ }^{115}$ S. Jabeen, ${ }^{115}$ G. Kukartsev, ${ }^{115}$ E. Laird, ${ }^{115}$ G. Landsberg, ${ }^{115}$ M. Luk, ${ }^{115}$ M. Narain, ${ }^{115}$ D. Nguyen, ${ }^{115}$ M. Segala, ${ }^{115}$ T. Sinthuprasith, ${ }^{115}$ T. Speer, ${ }^{115}$ K. V. Tsang,${ }^{115}$ R. Breedon,,${ }^{116}$ G. Breto, ${ }^{116}$ M. Calderon De La Barca Sanchez, ${ }^{116} \mathrm{~S}$. Chauhan, ${ }^{116}$ M. Chertok,${ }^{116}$ J. Conway, ${ }^{116}$ R. Conway, ${ }^{116}$ P. T. Cox, ${ }^{116}$ J. Dolen, ${ }^{116}$ R. Erbacher, ${ }^{16}$ M. Gardner, ${ }^{116}$ R. Houtz, ${ }^{116}$ W. Ko, ${ }^{116}$ A. Kopecky, ${ }^{16}$ R. Lander, ${ }^{16}$ T. Miceli, ${ }^{116}$ D. Pellett, ${ }^{116}$ F. Ricci-tam, ${ }^{116}$ B. Rutherford, ${ }^{116}$ M. Searle, ${ }^{116}$ J. Smith, ${ }^{116}$ M. Squires, ${ }^{116}$ M. Tripathi, ${ }^{116}$ R. Vasquez Sierra, ${ }^{116}$ V. Andreev, ${ }^{117}$ D. Cline,${ }^{117}$ R. Cousins,${ }^{117}$ J. Duris,${ }^{117}$ S. Erhan, ${ }^{117}$ P. Everaerts, ${ }^{117}$ C. Farrell, ${ }^{117}$ J. Hauser, ${ }^{117}$ M. Ignatenko, ${ }^{117}$ C. Jarvis, ${ }^{117}$ C. Plager, ${ }^{117}$ G. Rakness, ${ }^{117}$ P. Schlein, ${ }^{117, a}$ P. Traczyk,${ }^{117}$ 
V. Valuev, ${ }^{117}$ M. Weber, ${ }^{117}$ J. Babb, ${ }^{118}$ R. Clare, ${ }^{118}$ M. E. Dinardo, ${ }^{118}$ J. Ellison, ${ }^{118}$ J. W. Gary, ${ }^{118}$ F. Giordano, ${ }^{118}$ G. Hanson, ${ }^{118}$ G. Y. Jeng, ${ }^{118, z z}$ H. Liu, ${ }^{118}$ O. R. Long, ${ }^{118}$ A. Luthra, ${ }^{118}$ H. Nguyen, ${ }^{118}$ S. Paramesvaran, ${ }^{118}$ J. Sturdy, ${ }^{118}$ S. Sumowidagdo, ${ }^{118}$ R. Wilken, ${ }^{118}$ S. Wimpenny, ${ }^{118}$ W. Andrews, ${ }^{119}$ J. G. Branson, ${ }^{119}$ G. B. Cerati, ${ }^{119}$ S. Cittolin, ${ }^{119}$ D. Evans, ${ }^{119}$ F. Golf,${ }^{119}$ A. Holzner, ${ }^{119}$ R. Kelley ${ }^{119}$ M. Lebourgeois,${ }^{119}$ J. Letts, ${ }^{119}$ I. Macneill, ${ }^{119}$ B. Mangano, ${ }^{119}$ S. Padhi,${ }^{119}$ C. Palmer,${ }^{119}$ G. Petrucciani,${ }^{119}$ M. Pieri, ${ }^{119}$ M. Sani, ${ }^{119}$ V. Sharma, ${ }^{119}$ S. Simon, ${ }^{119}$ E. Sudano, ${ }^{119}$ M. Tadel, ${ }^{119}$ Y. Tu, ${ }^{119}$ A. Vartak, ${ }^{119}$ S. Wasserbaech, ${ }^{11, \text { aaa }}$ F. Würthwein, ${ }^{119}$ A. Yagil, ${ }^{119}$ J. Yoo, ${ }^{119}$ D. Barge, ${ }^{120}$ R. Bellan, ${ }^{120}$ C. Campagnari, ${ }^{120}$ M. D'Alfonso, ${ }^{120}$ T. Danielson, ${ }^{120}$ K. Flowers,${ }^{120}$ P. Geffert, ${ }^{120}$ J. Incandela,${ }^{120}$ C. Justus, ${ }^{120}$ P. Kalavase, ${ }^{120}$ S. A. Koay ${ }^{120}$ D. Kovalskyi, ${ }^{120}$ V. Krutelyov, ${ }^{120}$ S. Lowette,${ }^{120}$ N. Mccoll, ${ }^{120}$ V. Pavlunin, ${ }^{120}$ F. Rebassoo, ${ }^{120}$ J. Ribnik, ${ }^{120}$ J. Richman, ${ }^{120}$ R. Rossin, ${ }^{120}$ D. Stuart, ${ }^{120}$ W. To, ${ }^{120}$ C. West ${ }^{120}$ A. Apresyan, ${ }^{121}$ A. Bornheim, ${ }^{121}$ Y. Chen, ${ }^{121}$ E. Di Marco, ${ }^{121}$ J. Duarte, ${ }^{121}$ M. Gataullin, ${ }^{121}$ Y. Ma, ${ }^{121}$ A. Mott, ${ }^{121}$ H. B. Newman, ${ }^{121}$ C. Rogan, ${ }^{121}$ M. Spiropulu, ${ }^{121, e}$ V. Timciuc, ${ }^{121}$ J. Veverka, ${ }^{121}$ R. Wilkinson, ${ }^{121}$ Y. Yang, ${ }^{121}$ R. Y. Zhu, ${ }^{121}$ B. Akgun, ${ }^{122}$ V. Azzolini, ${ }^{122}$ R. Carroll, ${ }^{122}$ T. Ferguson, ${ }^{122}$ Y. Iiyama, ${ }^{122}$ D. W. Jang, ${ }^{122}$ Y. F. Liu, ${ }^{122}$ M. Paulini, ${ }^{122}$ H. Vogel, ${ }^{122}$ I. Vorobiev, ${ }^{122}$ J. P. Cumalat, ${ }^{123}$ B. R. Drell, ${ }^{123}$ C. J. Edelmaier, ${ }^{123}$ W. T. Ford, ${ }^{123}$ A. Gaz, ${ }^{123}$ B. Heyburn, ${ }^{123}$ E. Luiggi Lopez, ${ }^{123}$ J. G. Smith, ${ }^{123}$ K. Stenson, ${ }^{123}$ K. A. Ulmer, ${ }^{123}$ S. R. Wagner, ${ }^{123}$ J. Alexander, ${ }^{124}$ A. Chatterjee, ${ }^{124}$ N. Eggert, ${ }^{124}$ L. K. Gibbons, ${ }^{124}$ B. Heltsley, ${ }^{124}$

A. Khukhunaishvili, ${ }^{124}$ B. Kreis, ${ }^{124}$ N. Mirman, ${ }^{124}$ G. Nicolas Kaufman, ${ }^{124}$ J. R. Patterson, ${ }^{124}$ A. Ryd, ${ }^{124}$ E. Salvati, ${ }^{124}$ W. Sun, ${ }^{124}$ W. D. Teo, ${ }^{124}$ J. Thom, ${ }^{124}$ J. Thompson, ${ }^{124}$ J. Tucker, ${ }^{124}$ J. Vaughan, ${ }^{124}$ Y. Weng, ${ }^{124}$ L. Winstrom, ${ }^{124}$ P. Wittich, ${ }^{124}$ D. Winn, ${ }^{125}$ S. Abdullin, ${ }^{126}$ M. Albrow, ${ }^{126}$ J. Anderson, ${ }^{126}$ L. A. T. Bauerdick, ${ }^{126}$ A. Beretvas, ${ }^{126}$ J. Berryhill, ${ }^{126}$ P. C. Bhat, ${ }^{126}$ I. Bloch, ${ }^{126}$ K. Burkett ${ }^{126}$ J. N. Butler, ${ }^{126}$ V. Chetluru, ${ }^{126}$ H. W. K. Cheung, ${ }^{126}$ F. Chlebana, ${ }^{126}$ V. D. Elvira, ${ }^{126}$ I. Fisk, ${ }^{126}$ J. Freeman, ${ }^{126}$ Y. Gao, ${ }^{126}$ D. Green, ${ }^{126}$ O. Gutsche, ${ }^{126}$ J. Hanlon, ${ }^{126}$ R. M. Harris, ${ }^{126}$ J. Hirschauer, ${ }^{126}$ B. Hooberman, ${ }^{126}$ S. Jindariani, ${ }^{126}$ M. Johnson, ${ }^{126}$ U. Joshi, ${ }^{126}$ B. Kilminster, ${ }^{126}$ B. Klima, ${ }^{126}$ S. Kunori, ${ }^{126}$ S. Kwan, ${ }^{126}$ C. Leonidopoulos, ${ }^{126}$ J. Linacre, ${ }^{126}$ D. Lincoln, ${ }^{126}$ R. Lipton, ${ }^{126}$ J. Lykken, ${ }^{126}$ K. Maeshima, ${ }^{126}$ J. M. Marraffino, ${ }^{126}$ S. Maruyama, ${ }^{126}$ D. Mason, ${ }^{126}$ P. McBride, ${ }^{126}$ K. Mishra, ${ }^{126}$ S. Mrenna, ${ }^{126}$ Y. Musienko, ${ }^{126, b b b}$ C. Newman-Holmes, ${ }^{126}$ V. O’Dell, ${ }^{126}$ O. Prokofyev, ${ }^{126}$

E. Sexton-Kennedy, ${ }^{126}$ S. Sharma, ${ }^{126}$ W. J. Spalding, ${ }^{126}$ L. Spiegel, ${ }^{126}$ P. Tan, ${ }^{126}$ L. Taylor, ${ }^{126}$ S. Tkaczyk,${ }^{126}$ N. V. Tran, ${ }^{126}$ L. Uplegger, ${ }^{126}$ E. W. Vaandering, ${ }^{126}$ R. Vidal, ${ }^{126}$ J. Whitmore, ${ }^{126}$ W. Wu, ${ }^{126}$ F. Yang,,${ }^{126}$ F. Yumiceva, ${ }^{126}$ J. C. Yun, ${ }^{126}$ D. Acosta, ${ }^{127}$ P. Avery, ${ }^{127}$ D. Bourilkov, ${ }^{127}$ M. Chen, ${ }^{127}$ T. Cheng, ${ }^{127}$ S. Das, ${ }^{127}$ M. De Gruttola, ${ }^{127}$ G. P. Di Giovanni, ${ }^{127}$ D. Dobur, ${ }^{127}$ A. Drozdetskiy, ${ }^{127}$ R. D. Field, ${ }^{127}$ M. Fisher, ${ }^{127}$ Y. Fu, ${ }^{127}$

I. K. Furic, ${ }^{127}$ J. Gartner, ${ }^{127}$ J. Hugon, ${ }^{127}$ B. Kim, ${ }^{127}$ J. Konigsberg, ${ }^{127}$ A. Korytov, ${ }^{127}$ A. Kropivnitskaya, ${ }^{127}$

T. Kypreos, ${ }^{127}$ J. F. Low, ${ }^{127}$ K. Matchev ${ }^{127}$ P. Milenovic, ${ }^{127, c c c}$ G. Mitselmakher ${ }^{127}$ L. Muniz, ${ }^{127}$ R. Remington, ${ }^{127}$ A. Rinkevicius, ${ }^{127}$ P. Sellers, ${ }^{127}$ N. Skhirtladze, ${ }^{127}$ M. Snowball, ${ }^{127}$ J. Yelton, ${ }^{127}$ M. Zakaria, ${ }^{127}$ V. Gaultney, ${ }^{128}$ S. Hewamanage, ${ }^{128}$ L. M. Lebolo, ${ }^{128}$ S. Linn, ${ }^{128}$ P. Markowitz,${ }^{128}$ G. Martinez, ${ }^{128}$ J. L. Rodriguez, ${ }^{128}$ T. Adams, ${ }^{129}$ A. Askew, ${ }^{129}$ J. Bochenek, ${ }^{129}$ J. Chen, ${ }^{129}$ B. Diamond, ${ }^{129}$ S. V. Gleyzer, ${ }^{129}$ J. Haas, ${ }^{129}$ S. Hagopian, ${ }^{129}$ V. Hagopian, ${ }^{129}$ M. Jenkins, ${ }^{129}$ K. F. Johnson, ${ }^{129}$ H. Prosper, ${ }^{129}$ V. Veeraraghavan, ${ }^{129}$ M. Weinberg, ${ }^{129}$ M. M. Baarmand, ${ }^{130}$ B. Dorney, ${ }^{130}$ M. Hohlmann, ${ }^{130}$ H. Kalakhety, ${ }^{130}$ I. Vodopiyanov, ${ }^{130}$ M. R. Adams,,${ }^{131}$ I. M. Anghel, ${ }^{131}$ L. Apanasevich, ${ }^{131}$ Y. Bai, ${ }^{131}$ V. E. Bazterra, ${ }^{131}$ R. R. Betts, ${ }^{131}$ I. Bucinskaite, ${ }^{131}$ J. Callner, ${ }^{131}$ R. Cavanaugh, ${ }^{131}$ C. Dragoiu, ${ }^{131}$ O. Evdokimov, ${ }^{131}$ L. Gauthier, ${ }^{131}$ C. E. Gerber, ${ }^{131}$ D. J. Hofman, ${ }^{131}$ S. Khalatyan, ${ }^{131}$ F. Lacroix, ${ }^{131}$ M. Malek, ${ }^{131}$ C. O'Brien, ${ }^{131}$ C. Silkworth, ${ }^{131}$ D. Strom, ${ }^{131}$ N. Varelas, ${ }^{131}$ U. Akgun, ${ }^{132}$ E. A. Albayrak, ${ }^{132}$ B. Bilki, ${ }^{132, \text { ddd }}$ W. Clarida, ${ }^{132}$ F. Duru, ${ }^{132}$ S. Griffiths, ${ }^{132}$ J.-P. Merlo, ${ }^{132}$ H. Mermerkaya, ${ }^{132, \text { eee }}$ A. Mestvirishvili, ${ }^{132}$ A. Moeller, ${ }^{132}$ J. Nachtman, ${ }^{132}$ C. R. Newsom, ${ }^{132}$ E. Norbeck, ${ }^{132}$ Y. Onel, ${ }^{132}$ F. Ozok, ${ }^{132}$ S. Sen, ${ }^{132}$ E. Tiras, ${ }^{132}$ J. Wetzel,${ }^{132}$ T. Yetkin,${ }^{132}$ K. Yi, ${ }^{132}$ B. A. Barnett,,${ }^{133}$ B. Blumenfeld, ${ }^{133}$ S. Bolognesi, ${ }^{133}$ D. Fehling, ${ }^{133}$ G. Giurgiu, ${ }^{133}$ A. V. Gritsan, ${ }^{133}$ Z. J. Guo,${ }^{133}$ G. Hu, ${ }^{133}$ P. Maksimovic,,${ }^{133}$ S. Rappoccio, ${ }^{133}$ M. Swartz, ${ }^{133}$ A. Whitbeck, ${ }^{133}$ P. Baringer, ${ }^{134}$ A. Bean, ${ }^{134}$ G. Benelli, ${ }^{134}$ O. Grachov, ${ }^{134}$ R.P. Kenny Iii, ${ }^{134}$ M. Murray, ${ }^{134}$ D. Noonan, ${ }^{134}$ S. Sanders, ${ }^{134}$ R. Stringer,${ }^{134}$ G. Tinti, ${ }^{134}$ J. S. Wood, ${ }^{134}$ V. Zhukova, ${ }^{134}$ A. F. Barfuss, ${ }^{135}$ T. Bolton, ${ }^{135}$ I. Chakaberia, ${ }^{135}$ A. Ivanov, ${ }^{135}$ S. Khalil,${ }^{135}$ M. Makouski, ${ }^{135}$ Y. Maravin, ${ }^{135}$ S. Shrestha, ${ }^{135}$ I. Svintradze, ${ }^{135}$ J. Gronberg, ${ }^{136}$ D. Lange, ${ }^{136}$ D. Wright, ${ }^{136}$ A. Baden,,${ }^{137}$ M. Boutemeur, ${ }^{137}$ B. Calvert, ${ }^{137}$ S. C. Eno, ${ }^{137}$ J. A. Gomez, ${ }^{137}$ N. J. Hadley, ${ }^{137}$ R. G. Kellogg, ${ }^{137}$ M. Kirn,${ }^{137}$ T. Kolberg, ${ }^{137}$ Y. Lu, ${ }^{137}$ M. Marionneau, ${ }^{137}$ A. C. Mignerey, ${ }^{137}$ K. Pedro, ${ }^{137}$ A. Peterman, ${ }^{137}$ A. Skuja, ${ }^{137}$ J. Temple, ${ }^{137}$ M. B. Tonjes, ${ }^{137}$ S. C. Tonwar, ${ }^{137}$ E. Twedt, ${ }^{137}$ A. Apyan, ${ }^{138}$ G. Bauer, ${ }^{138}$ J. Bendavid, ${ }^{138}$ W. Busza, ${ }^{138}$ E. Butz, ${ }^{138}$ I. A. Cali, ${ }^{138}$ M. Chan, ${ }^{138}$ V. Dutta, ${ }^{138}$ G. Gomez Ceballos, ${ }^{138}$ M. Goncharov,${ }^{138}$ K. A. Hahn, ${ }^{138}$ Y. Kim, ${ }^{138}$ M. Klute, ${ }^{138}$ K. Krajczar, ${ }^{138, f f f}$ W. Li,${ }^{138}$ P. D. Luckey,${ }^{138}$ T. Ma, ${ }^{138}$ S. Nahn, ${ }^{138}$ C. Paus,${ }^{138}$ D. Ralph,${ }^{138}$ 
C. Roland, ${ }^{138}$ G. Roland, ${ }^{138}$ M. Rudolph, ${ }^{138}$ G. S. F. Stephans, ${ }^{138}$ F. Stöckli, ${ }^{138}$ K. Sumorok, ${ }^{138}$ K. Sung, ${ }^{138}$ D. Velicanu, ${ }^{138}$ E. A. Wenger, ${ }^{138}$ R. Wolf, ${ }^{138}$ B. Wyslouch,${ }^{138}$ S. Xie, ${ }^{138}$ M. Yang, ${ }^{138}$ Y. Yilmaz ${ }^{138}$ A. S. Yoon, ${ }^{138}$ M. Zanetti, ${ }^{138}$ S. I. Cooper, ${ }^{139}$ B. Dahmes, ${ }^{139}$ A. De Benedetti, ${ }^{139}$ G. Franzoni, ${ }^{139}$ A. Gude, ${ }^{139}$ S. C. Kao, ${ }^{139}$ K. Klapoetke, ${ }^{139}$ Y. Kubota, ${ }^{139}$ J. Mans, ${ }^{139}$ N. Pastika, ${ }^{139}$ R. Rusack, ${ }^{139}$ M. Sasseville, ${ }^{139}$ A. Singovsky, ${ }^{139}$ N. Tambe, ${ }^{139}$ J. Turkewitz, ${ }^{139}$ L. M. Cremaldi, ${ }^{140}$ R. Kroeger, ${ }^{140}$ L. Perera, ${ }^{140}$ R. Rahmat, ${ }^{140}$ D. A. Sanders, ${ }^{140}$ E. Avdeeva, ${ }^{141}$ K. Bloom, ${ }^{141}$ S. Bose,${ }^{141}$ J. Butt, ${ }^{141}$ D. R. Claes,${ }^{141}$ A. Dominguez,${ }^{141}$ M. Eads,${ }^{141}$ J. Keller, ${ }^{141}$ I. Kravchenko, ${ }^{141}$ J. Lazo-Flores, ${ }^{141}$ H. Malbouisson, ${ }^{141}$ S. Malik, ${ }^{141}$ G. R. Snow, ${ }^{141}$ U. Baur, ${ }^{142}$ A. Godshalk, ${ }^{142}$ I. Iashvili, ${ }^{142}$ S. Jain, ${ }^{142}$ A. Kharchilava, ${ }^{142}$ A. Kumar, ${ }^{142}$ S. P. Shipkowski, ${ }^{142}$ K. Smith, ${ }^{142}$ G. Alverson,,${ }^{143}$ E. Barberis, ${ }^{143}$ D. Baumgartel, ${ }^{143}$ M. Chasco, ${ }^{143}$ J. Haley,${ }^{143}$ D. Nash,${ }^{143}$ D. Trocino, ${ }^{143}$ D. Wood, ${ }^{143}$ J. Zhang, ${ }^{143}$ A. Anastassov, ${ }^{144}$ A. Kubik, ${ }^{144}$ N. Mucia, ${ }^{144}$ N. Odell, ${ }^{144}$ R. A. Ofierzynski, ${ }^{144}$ B. Pollack, ${ }^{144}$ A. Pozdnyakov, ${ }^{144}$ M. Schmitt, ${ }^{144}$ S. Stoynev, ${ }^{144}$ M. Velasco,${ }^{144}$ S. Won, ${ }^{144}$ L. Antonelli, ${ }^{145}$ D. Berry, ${ }^{145}$ A. Brinkerhoff, ${ }^{145}$ M. Hildreth, ${ }^{145}$ C. Jessop, ${ }^{145}$ D. J. Karmgard,${ }^{145}$ J. Kolb, ${ }^{145}$ K. Lannon, ${ }^{145}$ W. Luo, ${ }^{145}$ S. Lynch, ${ }^{145}$ N. Marinelli, ${ }^{145}$ D. M. Morse,${ }^{145}$ T. Pearson, ${ }^{145}$ R. Ruchti, ${ }^{145}$ J. Slaunwhite, ${ }^{145}$ N. Valls, ${ }^{145}$ M. Wayne, ${ }^{145}$ M. Wolf, ${ }^{145}$ B. Bylsma, ${ }^{146}$ L. S. Durkin, ${ }^{146}$ C. Hill,${ }^{146}$ R. Hughes, ${ }^{146}$ R. Hughes, ${ }^{146}$ K. Kotov, ${ }^{146}$ T. Y. Ling, ${ }^{146}$ D. Puigh,,${ }^{146}$ M. Rodenburg, ${ }^{146}$ C. Vuosalo, ${ }^{146}$ G. Williams, ${ }^{146}$ B. L. Winer, ${ }^{146}$ N. Adam, ${ }^{147}$ E. Berry, ${ }^{147}$ P. Elmer, ${ }^{147}$ D. Gerbaudo, ${ }^{147}$ V. Halyo, ${ }^{147}$ P. Hebda,${ }^{147}$ J. Hegeman, ${ }^{147}$ A. Hunt, ${ }^{147}$ P. Jindal, ${ }^{147}$ D. Lopes Pegna, ${ }^{147}$ P. Lujan, ${ }^{147}$ D. Marlow, ${ }^{147}$

T. Medvedeva, ${ }^{147}$ M. Mooney, ${ }^{147}$ J. Olsen, ${ }^{147}$ P. Piroué, ${ }^{147}$ X. Quan, ${ }^{147}$ A. Raval, ${ }^{147}$ B. Safdi, ${ }^{147}$ H. Saka, ${ }^{147}$ D. Stickland, ${ }^{147}$ C. Tully, ${ }^{147}$ J. S. Werner, ${ }^{147}$ A. Zuranski, ${ }^{147}$ J. G. Acosta, ${ }^{148}$ E. Brownson, ${ }^{148}$ X. T. Huang, ${ }^{148}$ A. Lopez, ${ }^{148}$ H. Mendez, ${ }^{148}$ S. Oliveros, ${ }^{148}$ J.E. Ramirez Vargas, ${ }^{148}$ A. Zatserklyaniy, ${ }^{148}$ E. Alagoz, ${ }^{149}$ V.E. Barnes, ${ }^{149}$ D. Benedetti, ${ }^{149}$ G. Bolla, ${ }^{149}$ D. Bortoletto, ${ }^{149}$ M. De Mattia, ${ }^{149}$ A. Everett, ${ }^{149}$ Z. Hu, ${ }^{149}$ M. Jones, ${ }^{149}$ O. Koybasi, ${ }^{149}$ M. Kress, ${ }^{149}$ A. T. Laasanen, ${ }^{149}$ N. Leonardo, ${ }^{149}$ V. Maroussov, ${ }^{149}$ P. Merkel, ${ }^{149}$ D. H. Miller,${ }^{149}$ N. Neumeister, ${ }^{149}$ I. Shipsey, ${ }^{149}$ D. Silvers, ${ }^{149}$ A. Svyatkovskiy, ${ }^{149}$ M. Vidal Marono, ${ }^{149}$ H. D. Yoo, ${ }^{149}$ J. Zablocki, ${ }^{149}$ Y. Zheng, ${ }^{149}$ S. Guragain, ${ }^{150}$ N. Parashar, ${ }^{150}$ A. Adair, ${ }^{151}$ C. Boulahouache, ${ }^{151}$ K. M. Ecklund, ${ }^{151}$ F. J. M. Geurts, ${ }^{151}$ B. P. Padley, ${ }^{151}$ R. Redjimi, ${ }^{151}$ J. Roberts, ${ }^{151}$ J. Zabel, ${ }^{151}$ B. Betchart,${ }^{152}$ A. Bodek, ${ }^{152}$ Y. S. Chung, ${ }^{152}$ R. Covarelli, ${ }^{152}$ P. de Barbaro, ${ }^{152}$ R. Demina, ${ }^{152}$ Y. Eshaq, ${ }^{152}$ A. Garcia-Bellido, ${ }^{152}$ P. Goldenzweig, ${ }^{152}$ J. Han, ${ }^{152}$ A. Harel, ${ }^{152}$ D. C. Miner ${ }^{152}$ D. Vishnevskiy, ${ }^{152}$ M. Zielinski, ${ }^{152}$ A. Bhatti, ${ }^{153}$ R. Ciesielski, ${ }^{153}$ L. Demortier, ${ }^{153}$ K. Goulianos,${ }^{153}$ G. Lungu, ${ }^{153}$ S. Malik, ${ }^{153}$ C. Mesropian, ${ }^{153}$ S. Arora, ${ }^{154}$ A. Barker, ${ }^{154}$ J. P. Chou, ${ }^{154}$ C. Contreras-Campana, ${ }^{154}$ E. Contreras-Campana, ${ }^{154}$ D. Duggan, ${ }^{154}$ D. Ferencek, ${ }^{154}$ Y. Gershtein, ${ }^{154}$ R. Gray, ${ }^{154}$ E. Halkiadakis, ${ }^{154}$ D. Hidas, ${ }^{154}$ A. Lath, ${ }^{154}$ S. Panwalkar, ${ }^{154}$ M. Park, ${ }^{154}$ R. Patel, ${ }^{154}$ V. Rekovic,,${ }^{154}$ J. Robles,,${ }^{154}$ K. Rose,,${ }^{154}$ S. Salur, ${ }^{154}$ S. Schnetzer, ${ }^{154}$ C. Seitz, ${ }^{154}$ S. Somalwar, ${ }^{154}$ R. Stone, ${ }^{154}$ S. Thomas, ${ }^{154}$ G. Cerizza, ${ }^{155}$ M. Hollingsworth, ${ }^{155}$ S. Spanier, ${ }^{155}$ Z. C. Yang, ${ }^{155}$ A. York, ${ }^{155}$ R. Eusebi, ${ }^{156}$ W. Flanagan, ${ }^{156}$ J. Gilmore, ${ }^{156}$ T. Kamon, ${ }^{156, g g g}$ V. Khotilovich, ${ }^{156}$ R. Montalvo, ${ }^{156}$ I. Osipenkov, ${ }^{156}$ Y. Pakhotin, ${ }^{156}$ A. Perloff, ${ }^{156}$ J. Roe, ${ }^{156}$ A. Safonov, ${ }^{156}$ T. Sakuma, ${ }^{156}$ S. Sengupta, ${ }^{156}$ I. Suarez, ${ }^{156}$ A. Tatarinov, ${ }^{156}$ D. Toback, ${ }^{156}$ N. Akchurin, ${ }^{157}$ J. Damgov, ${ }^{157}$ P. R. Dudero, ${ }^{157}$ C. Jeong, ${ }^{157}$ K. Kovitanggoon, ${ }^{157}$ S. W. Lee, ${ }^{157}$ T. Libeiro, ${ }^{157}$ Y. Roh, ${ }^{157}$ I. Volobouev, ${ }^{157}$ E. Appelt, ${ }^{158}$ A. G. Delannoy, ${ }^{158}$ C. Florez, ${ }^{158}$ S. Greene, ${ }^{158}$ A. Gurrola, ${ }^{158}$ W. Johns, ${ }^{158}$ C. Johnston, ${ }^{158}$ P. Kurt, ${ }^{158}$ C. Maguire, ${ }^{158}$ A. Melo, ${ }^{158}$ M. Sharma, ${ }^{158}$ P. Sheldon, ${ }^{158}$ B. Snook,${ }^{158}$ S. Tuo,${ }^{158}$ J. Velkovska, ${ }^{158}$ M. W. Arenton, ${ }^{159}$ M. Balazs, ${ }^{159}$ S. Boutle ${ }^{159}$ B. Cox,${ }^{159}$ B. Francis,${ }^{159}$ J. Goodell, ${ }^{159}$ R. Hirosky, ${ }^{159}$ A. Ledovskoy, ${ }^{159}$ C. Lin, ${ }^{159}$ C. Neu, ${ }^{159}$ J. Wood, ${ }^{159}$ R. Yohay, ${ }^{159}$ S. Gollapinni, ${ }^{160}$ R. Harr, ${ }^{160}$ P. E. Karchin, ${ }^{160}$

C. Kottachchi Kankanamge Don, ${ }^{160}$ P. Lamichhane, ${ }^{160}$ A. Sakharov ${ }^{160}$ M. Anderson, ${ }^{161}$ M. Bachtis,${ }^{161}$ D. Belknap, ${ }^{161}$ L. Borrello, ${ }^{161}$ D. Carlsmith,${ }^{161}$ M. Cepeda, ${ }^{161}$ S. Dasu, ${ }^{161}$ E. Friis, ${ }^{161}$ L. Gray, ${ }^{161}$ K. S. Grogg, ${ }^{161}$ M. Grothe, ${ }^{161}$ R. Hall-Wilton, ${ }^{161}$ M. Herndon, ${ }^{161}$ A. Hervé, ${ }^{161}$ P. Klabbers, ${ }^{161}$ J. Klukas, ${ }_{161}$ A. Lanaro, ${ }^{161}$ C. Lazaridis, ${ }^{161}$ J. Leonard, ${ }^{161}$ R. Loveless, ${ }^{161}$ A. Mohapatra, ${ }^{161}$ I. Ojalvo, ${ }^{161}$ F. Palmonari, ${ }^{161}$ G. A. Pierro, ${ }^{161}$ I. Ross, ${ }^{161}$ A. Savin, ${ }^{161}$ W. H. Smith, ${ }^{161}$ and J. Swanson ${ }^{161}$

(CMS Collaboration)

\author{
${ }^{1}$ Yerevan Physics Institute, Yerevan, Armenia \\ ${ }^{2}$ Institut für Hochenergiephysik der OeAW, Wien, Austria \\ ${ }^{3}$ National Centre for Particle and High Energy Physics, Minsk, Belarus \\ ${ }^{4}$ Universiteit Antwerpen, Antwerpen, Belgium \\ ${ }^{5}$ Vrije Universiteit Brussel, Brussel, Belgium \\ ${ }^{6}$ Université Libre de Bruxelles, Bruxelles, Belgium
}




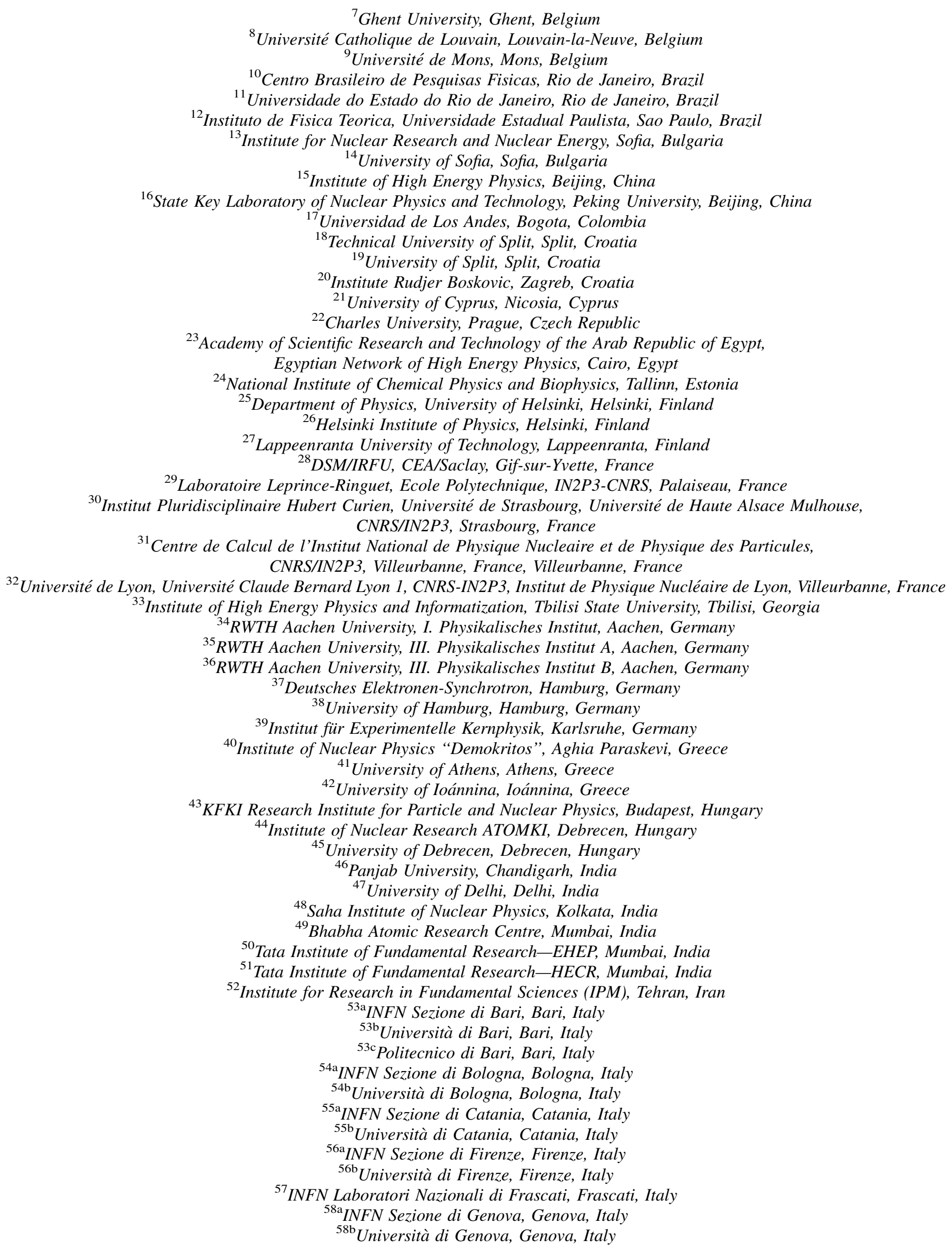


${ }^{59 a}$ INFN Sezione di Milano-Bicocca, Milano, Italy

${ }^{59 b}$ Università di Milano-Bicocca, Milano, Italy

${ }^{60 a}$ INFN Sezione di Napoli, Napoli, Italy

${ }^{60 \mathrm{~b}}$ Università di Napoli “Federico II”, Napoli, Italy

${ }^{61 a}$ INFN Sezione di Padova, Padova, Italy

${ }^{61 \mathrm{~b}}$ Università di Padova, Padova, Italy

${ }^{61 \mathrm{c}}$ Università di Trento (Trento), Padova, Italy

${ }^{62 a}$ INFN Sezione di Pavia, Pavia, Italy

${ }^{62 \mathrm{~b}}$ Università di Pavia, Pavia, Italy

${ }^{63 a}$ INFN Sezione di Perugia, Perugia, Italy

${ }^{63 \mathrm{~b}}$ Università di Perugia, Perugia, Italy

${ }^{64 a}$ INFN Sezione di Pisa, Pisa, Italy

${ }^{64 \mathrm{~b}}$ Università di Pisa, Pisa, Italy

${ }^{64 \mathrm{c}}$ Scuola Normale Superiore di Pisa, Pisa, Italy

${ }^{65}$ INFN Sezione di Roma, Roma, Italy

${ }^{65 b}$ Università di Roma “La Sapienza”, Roma, Italy

${ }^{66 \mathrm{a}}$ INFN Sezione di Torino, Torino, Italy

${ }^{66 \mathrm{~b}}$ Università di Torino, Torino, Italy

${ }^{66 c}$ Università del Piemonte Orientale (Novara), Torino, Italy

${ }^{67}$ INFN Sezione di Trieste, Trieste, Italy

${ }^{67 \mathrm{~b}}$ Università di Trieste, Trieste, Italy

${ }^{68}$ Kangwon National University, Chunchon, Korea

${ }^{69}$ Kyungpook National University, Daegu, Korea

${ }^{70}$ Chonnam National University, Institute for Universe and Elementary Particles, Kwangju, Korea

${ }^{71}$ Korea University, Seoul, Korea

${ }^{72}$ University of Seoul, Seoul, Korea

${ }^{73}$ Sungkyunkwan University, Suwon, Korea

${ }^{74}$ Vilnius University, Vilnius, Lithuania

${ }^{75}$ Centro de Investigacion y de Estudios Avanzados del IPN, Mexico City, Mexico

${ }^{76}$ Universidad Iberoamericana, Mexico City, Mexico

${ }^{77}$ Benemerita Universidad Autonoma de Puebla, Puebla, Mexico

${ }^{78}$ Universidad Autónoma de San Luis Potosí, San Luis Potosí, Mexico

${ }^{79}$ University of Auckland, Auckland, New Zealand

${ }^{80}$ University of Canterbury, Christchurch, New Zealand

${ }^{81}$ National Centre for Physics, Quaid-I-Azam University, Islamabad, Pakistan

${ }^{82}$ National Centre for Nuclear Research, Swierk, Poland

${ }^{83}$ Institute of Experimental Physics, Faculty of Physics, University of Warsaw, Warsaw, Poland

${ }^{84}$ Laboratório de Instrumentação e Física Experimental de Partículas, Lisboa, Portugal

${ }^{85}$ Joint Institute for Nuclear Research, Dubna, Russia

${ }^{86}$ Petersburg Nuclear Physics Institute, Gatchina (St. Petersburg), Russia

${ }^{87}$ Institute for Nuclear Research, Moscow, Russia

${ }^{88}$ Institute for Theoretical and Experimental Physics, Moscow, Russia

${ }^{89}$ Moscow State University, Moscow, Russia

${ }^{90}$ P.N. Lebedev Physical Institute, Moscow, Russia

${ }^{91}$ State Research Center of Russian Federation, Institute for High Energy Physics, Protvino, Russia

${ }^{92}$ University of Belgrade, Faculty of Physics and Vinca Institute of Nuclear Sciences, Belgrade, Serbia

${ }^{93}$ Centro de Investigaciones Energéticas Medioambientales y Tecnológicas (CIEMAT), Madrid, Spain

${ }^{94}$ Universidad Autónoma de Madrid, Madrid, Spain

${ }^{95}$ Universidad de Oviedo, Oviedo, Spain

${ }^{96}$ Instituto de Física de Cantabria (IFCA), CSIC-Universidad de Cantabria, Santander, Spain

${ }^{97}$ CERN, European Organization for Nuclear Research, Geneva, Switzerland

${ }^{98}$ Paul Scherrer Institut, Villigen, Switzerland

${ }^{99}$ Institute for Particle Physics, ETH Zurich, Zurich, Switzerland

${ }^{100}$ Universität Zürich, Zurich, Switzerland

${ }^{101}$ National Central University, Chung-Li, Taiwan

${ }^{102}$ National Taiwan University (NTU), Taipei, Taiwan

${ }^{103}$ Cukurova University, Adana, Turkey

${ }^{104}$ Middle East Technical University, Physics Department, Ankara, Turkey

${ }^{105}$ Bogazici University, Istanbul, Turkey

${ }^{106}$ Istanbul Technical University, Istanbul, Turkey

${ }^{107}$ National Scientific Center, Kharkov Institute of Physics and Technology, Kharkov, Ukraine 
${ }^{108}$ University of Bristol, Bristol, United Kingdom

${ }^{109}$ Rutherford Appleton Laboratory, Didcot, United Kingdom

${ }^{110}$ Imperial College, London, United Kingdom

${ }^{111}$ Brunel University, Uxbridge, United Kingdom

${ }^{112}$ Baylor University, Waco, Texas, USA

${ }^{113}$ The University of Alabama, Tuscaloosa, Alabama, USA

${ }^{114}$ Boston University, Boston, Massachusetts, USA

${ }^{115}$ Brown University, Providence, Rhode Island, USA

${ }^{116}$ University of California, Davis, Davis, California, USA

${ }^{117}$ University of California, Los Angeles, Los Angeles, California, USA

${ }^{118}$ University of California, Riverside, Riverside, California, USA

${ }^{119}$ University of California, San Diego, La Jolla, California, USA

${ }^{120}$ University of California, Santa Barbara, Santa Barbara, California, USA

${ }^{121}$ California Institute of Technology, Pasadena, California, USA

${ }^{122}$ Carnegie Mellon University, Pittsburgh, Pennsylvania, USA

${ }^{123}$ University of Colorado at Boulder, Boulder, Colorado, USA

${ }^{124}$ Cornell University, Ithaca, New York, USA

${ }^{125}$ Fairfield University, Fairfield, Connecticut, USA

${ }^{126}$ Fermi National Accelerator Laboratory, Batavia, Illinois, USA

${ }^{127}$ University of Florida, Gainesville, Florida, USA

${ }^{128}$ Florida International University, Miami, Florida, USA

${ }^{129}$ Florida State University, Tallahassee, Florida, USA

${ }^{130}$ Florida Institute of Technology, Melbourne, Florida, USA

${ }^{131}$ University of Illinois at Chicago (UIC), Chicago, Illinois, USA

${ }^{132}$ The University of Iowa, Iowa City, Iowa, USA

${ }^{133}$ Johns Hopkins University, Baltimore, Maryland, USA

${ }^{134}$ The University of Kansas, Lawrence, Kansas, USA

${ }^{135}$ Kansas State University, Manhattan, Kansas, USA

${ }^{136}$ Lawrence Livermore National Laboratory, Livermore, California, USA

${ }^{137}$ University of Maryland, College Park, Maryland, USA

${ }^{138}$ Massachusetts Institute of Technology, Cambridge, Massachusetts, USA

${ }^{139}$ University of Minnesota, Minneapolis, Minnesota, USA

${ }^{140}$ University of Mississippi, University, Mississippi, USA

${ }^{141}$ University of Nebraska-Lincoln, Lincoln, Nebraska, USA

${ }^{142}$ State University of New York at Buffalo, Buffalo, New York, USA

${ }^{143}$ Northeastern University, Boston, Massachusetts, USA

${ }^{144}$ Northwestern University, Evanston, Illinois, USA

${ }^{145}$ University of Notre Dame, Notre Dame, Indiana, USA

${ }^{146}$ The Ohio State University, Columbus, Ohio, USA

${ }^{147}$ Princeton University, Princeton, New Jersey, USA

${ }^{148}$ University of Puerto Rico, Mayaguez, Puerto Rico, USA

${ }^{149}$ Purdue University, West Lafayette, Indiana, USA

${ }^{150}$ Purdue University Calumet, Hammond, Indiana, USA

${ }^{151}$ Rice University, Houston, Texas, USA

${ }^{152}$ University of Rochester, Rochester, New York, USA

${ }^{153}$ The Rockefeller University, New York, New York, USA

${ }^{154}$ Rutgers, the State University of New Jersey, Piscataway, New Jersey, USA

${ }^{155}$ University of Tennessee, Knoxville, Tennessee, USA

${ }^{156}$ Texas A\&M University, College Station, Texas, USA

${ }^{157}$ Texas Tech University, Lubbock, Texas, USA

${ }^{158}$ Vanderbilt University, Nashville, Tennessee, USA

${ }^{159}$ University of Virginia, Charlottesville, Virginia, USA

${ }^{160}$ Wayne State University, Detroit, Michigan, USA

${ }^{161}$ University of Wisconsin, Madison, Wisconsin, USA

${ }^{\mathrm{a}}$ Deceased.

${ }^{\mathrm{b}}$ Also at Vienna University of Technology, Vienna, Austria.

${ }^{\mathrm{c}}$ Also at National Institute of Chemical Physics and Biophysics, Tallinn, Estonia.

${ }^{\mathrm{d}}$ Also at Universidade Federal do ABC, Santo Andre, Brazil.

${ }^{\mathrm{e}}$ Also at California Institute of Technology, Pasadena, California, USA. 
${ }_{\mathrm{f}}^{\mathrm{f}}$ Also at CERN, European Organization for Nuclear Research, Geneva, Switzerland.

${ }^{\mathrm{g}}$ Also at Laboratoire Leprince-Ringuet, Ecole Polytechnique, IN2P3-CNRS, Palaiseau, France.

${ }^{\mathrm{h}}$ Also at Suez Canal University, Suez, Egypt.

${ }^{\mathrm{i}}$ Also at Zewail City of Science and Technology, Zewail, Egypt.

${ }^{\mathrm{j}}$ Also at Cairo University, Cairo, Egypt.

${ }^{\mathrm{k}}$ Also at Fayoum University, El-Fayoum, Egypt.

${ }^{1}$ Also at British University, Cairo, Egypt.

${ }^{\mathrm{m}}$ Now at Ain Shams University, Cairo, Egypt.

${ }^{\mathrm{n}}$ Also at National Centre for Nuclear Research, Swierk, Poland.

${ }^{\circ}$ Also at Université de Haute-Alsace, Mulhouse, France.

${ }^{\mathrm{p}}$ Now at Joint Institute for Nuclear Research, Dubna, Russia.

${ }^{\mathrm{q}}$ Also at Moscow State University, Moscow, Russia.

${ }^{\mathrm{r}}$ Also at Brandenburg University of Technology, Cottbus, Germany.

${ }^{s}$ Also at Institute of Nuclear Research ATOMKI, Debrecen, Hungary.

${ }^{\mathrm{t}}$ Also at Eötvös Loránd University, Budapest, Hungary.

${ }^{\mathrm{u}}$ Also at Tata Institute of Fundamental Research-HECR, Mumbai, India.

${ }^{\mathrm{v}}$ Also at University of Visva-Bharati, Santiniketan, India.

${ }^{w}$ Also at Sharif University of Technology, Tehran, Iran.

${ }^{\mathrm{x}}$ Also at Isfahan University of Technology, Isfahan, Iran.

${ }^{y}$ Also at Plasma Physics Research Center, Science and Research Branch, Islamic Azad University, Teheran, Iran.

${ }^{\mathrm{z}}$ Also at Facoltà Ingegneria Università di Roma, Roma, Italy.

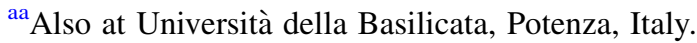

${ }^{\mathrm{bb}}$ Also at Università degli Studi Guglielmo Marconi, Roma, Italy.

${ }^{c c}$ Also at Laboratori Nazionali di Legnaro dell' INFN, Legnaro, Italy.

${ }^{\mathrm{dd}}$ Also at Università degli Studi di Siena, Siena, Italy.

${ }^{e e}$ Also at University of Bucharest, Faculty of Physics, Bucuresti-Magurele, Romania.

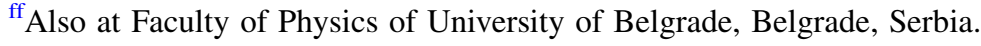

${ }^{g g}$ Also at University of California, Los Angeles, Los Angeles, California, USA.

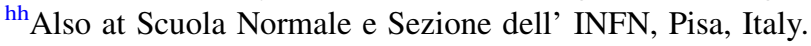

${ }^{\text {ii }}$ Also at INFN Sezione di Roma, Università di Roma "La Sapienza", Roma, Italy.

${ }^{\mathrm{jj}}$ Also at University of Athens, Athens, Greece.

${ }^{\mathrm{kk}}$ Also at Rutherford Appleton Laboratory, Didcot, United Kingdom.

${ }^{11}$ Also at The University of Kansas, Lawrence, Kansas, USA.

${ }^{\mathrm{mm}}$ Also at Paul Scherrer Institut, Villigen, Switzerland

${ }^{\mathrm{nn}}$ Also at Institute for Theoretical and Experimental Physics, Moscow, Russia.

${ }^{\circ}$ Also at Gaziosmanpasa University, Tokat, Turkey.

${ }^{\mathrm{pp}}$ Also at Adiyaman University, Adiyaman, Turkey.

${ }^{\mathrm{qq}} \mathrm{Also}$ at Izmir Institute of Technology, Izmir, Turkey.

${ }^{\mathrm{rr}}$ Also at The University of Iowa, Iowa City, Iowa, USA.

${ }^{\text {ss }}$ Also at Mersin University, Mersin, Turkey.

${ }^{t t}$ Also at Ozyegin University, Istanbul, Turkey.

${ }^{\mathrm{uu}}$ Also at Kafkas University, Kars, Turkey.

${ }^{\mathrm{vv}}$ Also at Suleyman Demirel University, Isparta, Turkey.

${ }^{\text {ww }}$ Also at Ege University, Izmir, Turkey.

${ }^{\mathrm{xx}}$ Also at School of Physics and Astronomy, University of Southampton, Southampton, United Kingdom.

${ }^{\text {yy } A l s o ~ a t ~ I N F N ~ S e z i o n e ~ d i ~ P e r u g i a, ~ U n i v e r s i t a ̀ ~ d i ~ P e r u g i a, ~ P e r u g i a, ~ I t a l y . ~}$

${ }^{\mathrm{zz}}$ Also at University of Sydney, Sydney, Australia.

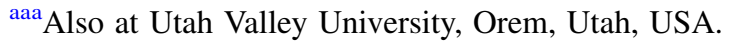

${ }^{\mathrm{bbb}}$ Also at Institute for Nuclear Research, Moscow, Russia.

${ }^{c c c}$ Also at University of Belgrade, Faculty of Physics and Vinca Institute of Nuclear Sciences, Belgrade, Serbia.

${ }^{\text {ddd }}$ Also at Argonne National Laboratory, Argonne, Illinois, USA.

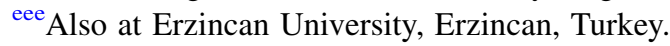

${ }^{\mathrm{fff}} \mathrm{Also}$ at KFKI Research Institute for Particle and Nuclear Physics, Budapest, Hungary.

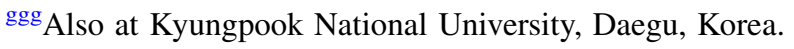

